\title{
A Complete Stability Analysis of Planar Linear Systems Under Saturation
}

\author{
Tingshu Hu, Student Member, IEEE, and Zongli Lin, Senior Member, IEEE
}

\begin{abstract}
A complete stability analysis is performed on a planar system of the form $\dot{x}=\sigma(A x)$ where $A$ is a Hurwitz matrix and $\sigma$ is the saturation function. Necessary and sufficient conditions for the system to be globally asymptotically stable (GAS) or to have a closed trajectory are explicitly given in terms of the entries of $A$. These conditions also indicate that the system always has a closed trajectory if it is not GAS.
\end{abstract}

Index Terms-Closed trajectories, neural networks, saturation, stability.

\section{INTRODUCTION}

D YNAMICAL systems with saturation nonlinearities arise frequently in neural networks, analog circuits, and control systems (see, for example, [2], [4], [5], and [8] and the references therein). In this paper, we consider the systems of the following form:

$$
\dot{x}=\sigma(A x), \quad x \in R^{n}
$$

where $\sigma: R^{n} \rightarrow R^{n}$ is the standard saturation function. With a slight abuse of notation, we use the same symbol to denote both the vector saturation function and the scalar saturation function, i.e., if $v \in \boldsymbol{R}^{n}$, then $\sigma(v)=\left[\sigma\left(v_{1}\right), \sigma\left(v_{2}\right), \cdots, \sigma\left(v_{n}\right)\right]^{T}$ and

$$
\sigma\left(v_{i}\right)= \begin{cases}-1, & \text { if } v_{i}<-1 \\ v_{i}, & \text { if }-1 \leq v_{i} \leq 1 \\ 1 & \text { if } v_{i}>1\end{cases}
$$

Systems of the form (1) and their discrete counterparts mainly arise in neural networks and in digital filters.

As with any dynamical system, stability of these systems is of primary concern and has been heavily studied in the literature for a long period of time (see, for example, [1], [6], [7], [8], and [10] and the references therein). As seen in the literature, the stability analysis of such systems are highly nontrivial. Even for the planar case, only sufficient conditions for global asymptotic stability are available [1], [8], [10]. In this paper, we present a complete analysis of the planar system of the form (1). In particular, necessary and sufficient conditions for the system to be globally asymptotically stable (GAS) or to have a limit circle are explicitly given in terms of the entries of the matrix $A$. We

Manuscript received April 14, 1999; revised September 15, 1999. This work was supported in part by the U.S. Office of Naval Research Young Investigator Program under Grant N00014-99-1-0670. This paper was recommended by Associate Editor V. Pérez Villar.

The authors are with the Department of Electrical Engineering, University of Virginia, Charlottesville, VA 22903 USA (e-mail: th7f@virginia.edu; zl5y@virginia.edu).

Publisher Item Identifier S 1057-7122(00)02918-4. will also describe a surprising, but appealing, phenomenon that even with an unstable matrix $A$ it is still possible for the system to have a bounded global attractor.

We would like to point out that the necessary and sufficient conditions for planar linear systems operating on the unit square to be GAS were recently identified in [8] and [9]. The class of linear systems operating on the unit square can be put in a form similar to (1) with $\sigma$ being a state-dependent function that takes zero value instead of \pm 1 as the saturation function does, whenever the state is to leave the unit square. By forcing the state within the unit square, the dynamical behavior is completely different. For example, the closed trajectory would not exist [9].

We will begin searching for the necessary and sufficient condition for the system to be GAS by drawing a general picture of the vector field in Section III. Some constants are captured to characterize the vector field. In Section IV, we show that it is these constants, rather than the stability of the $A$ matrix, that determine the global boundedness of the trajectories. An interesting example is presented to show that even if $A$ is unstable, the system can still have a bounded global attractor.

The condition for the existence of a bounded global attractor as given in Section IV, along with the stability of the matrix $A$, guarantees the system to be GAS. This is shown in Section V. Now that all the trajectories are bounded, the only problem to be solved in Section V is the nonexistence of a closed trajectory. This problem turns out to be quite complicated due to the partition of the vector field by the saturation. In the central unit square $\sigma(x)=x$, and a trajectory in this region follows that of a linear system. Off the central square, the sequence of the intersections of a trajectory with a straight line is governed by a first-order linear time invariant discrete-time system. The real complexity arises when a trajectory traverses between the central square and other regions. We will approach this problem through evolving models with

$$
A=\left[\begin{array}{cc}
-1, & a_{12} \\
-k a_{12} a_{22}, & a_{22}
\end{array}\right], \quad a_{12}>1, k \geq 1 .
$$

In the primary model $a_{22}=1$ and $k=1$. In the secondary model $a_{22}=1$ and $k \geq 1$. In the third-level model $a_{22} \in$ $(0,1)$ and $k \geq 1$. The trajectories of the secondary model are very appealing. Inside a certain ellipse all the trajectories are closed and outside this ellipse all the trajectories converge to this ellipse. We will establish our main results by comparing the trajectories of the general model with those of a secondary model, which in turn are characterized by comparing with the primary model. 


\section{MAIN RESULTS}

Consider the following system

$$
\dot{x}=\sigma(A x), \quad x \in R^{2}
$$

where $\sigma: \boldsymbol{R}^{2} \rightarrow \boldsymbol{R}^{2}$ is the saturation function, i.e., if $v \in \boldsymbol{R}^{2}$, then $\sigma(v)=\left[\begin{array}{l}\sigma\left(v_{1}\right) \\ \sigma\left(v_{2}\right)\end{array}\right]$ and $\sigma$ is as defined by (2). For this system, we observe that its dynamics are left unchanged under the state transformation $z=T x$ if $T$ is of the form $T=P S$ where $P$ is a permutation matrix and $S=\operatorname{diag}( \pm 1, \pm 1)$.

We assume through out this paper that $\operatorname{det}(A) \neq 0$. This implies that $A$ is nonsingular and the system has a unique equilibrium point at the origin. Following the idea of [1], let $z=A x$. The system (3) is then transformed into the following form:

$$
\dot{z}=A \dot{x}=A \sigma(A x)=A \sigma(z) .
$$

We see that the dynamics of the system (3) and hence its stability properties are equivalent to those of the system

$$
\dot{x}=A \sigma(x) \text {. }
$$

We will focus on (4) in this paper.

Given an initial state $x_{0}$, denote the trajectory of the system (4) that passes through $x_{0}$ at $t=0$ as $\psi\left(t, x_{0}\right)$. Mainly, we consider the positive trajectory $\psi\left(t, x_{0}\right), t \geq 0$. However, occasionally we use $\psi\left(-t, x_{0}\right), t \geq 0$ for the purpose of comparison.

Definition 2.1: The system (4) is said to be stable at its equilibrium $x_{e}=0$ if, for any $\varepsilon>0$, there exists a $\delta>0$ such that $\left\|\psi\left(t, x_{0}\right)\right\| \leq \varepsilon$ for all $t \geq 0$ and $\left\|x_{0}\right\| \leq \delta$. It is said to be GAS if $x_{e}=0$ is a stable equilibrium and $\lim _{t \rightarrow \infty} \psi\left(t, x_{0}\right)=0$ for all $x_{0} \in R^{2}$. Also, it is said to be locally asymptotically stable if it is stable and $\lim _{t \rightarrow \infty} \psi\left(t, x_{0}\right)=0$ for $x_{0} \in U_{0}$, a neighborhood of $x_{e}=0$.

Obviously, $x_{e}=0$ is a locally asymptotically stable equilibrium if and only if $A$ is Hurwitz. In this case, at least one of its diagonal elements must be negative. Without loss of generality, we assume throughout the remaining part of this paper that

$$
A=\left[\begin{array}{ll}
-a_{11}, & a_{12} \\
-a_{21}, & a_{22}
\end{array}\right], \quad a_{11}>0, a_{21} \geq 0 .
$$

Otherwise, we can use $T=\left[\begin{array}{ll}0 & 1 \\ 1 & 0\end{array}\right]$ as the state transformation matrix to make $a_{11}>0$ or use $T=\left[\begin{array}{ll}1 & -0 \\ 0 & -1\end{array}\right]$ to make $a_{21} \geq 0$.

Our main result in this paper, presented in the following theorem, gives a complete description of the stability properties of the system (4) with $A$ given in (5). As explained above, any Hurwitz $A$ can be transformed into the form of (5).

Theorom 2.1: The system (4) is GAS if and only if $A$ is Hurwitz and one of the following conditions is satisfied:

a) $a_{22}<0$;

b) $a_{22} \geq 0$ and $a_{11} a_{21} \geq a_{12} a_{22}$.

On the other hand, if none of a) and b) is satisfied, the system will have diverging trajectories and there will be a closed trajectory.

In proving this main result, we will also obtain conditions under which all the trajectories of the system (4) are bounded.
Remark 2.1: We recall a recent sufficient condition for global asymptotic stability of the system (4) from [1]. The results of [1], tailored to the special form of $A$ in (5), is summarized as follows. the system (4) is GAS if $A$ is Hurwitz and one of the following conditions is satisfied.

a) $a_{22}<0$.

b) $a_{22} \geq 0$ and $a_{11}>a_{12}$.

The fact that $a_{22} \geq 0$ and $A$ is Hurwitz imply that $a_{12}>0$.

In view of Remark 2.1, we only need to consider the case where $a_{22} \geq 0$ and $a_{11} \leq a_{12}$. In this case, the four parameters $a_{11}, a_{12}, a_{21}, a_{22}$ are all nonnegative.

\section{THE VECTOR FIELD}

In this section, we present a general picture of the following vector field:

$$
\begin{aligned}
& \dot{x}_{1}=-a_{11} \sigma\left(x_{1}\right)+a_{12} \sigma\left(x_{2}\right)=: f_{1}(x), \\
& \dot{x}_{2}=-a_{21} \sigma\left(x_{1}\right)+a_{22} \sigma\left(x_{2}\right)=: f_{2}(x)
\end{aligned}
$$

where $0<a_{11} \leq a_{12}, a_{21}, a_{22} \geq 0$ and $\operatorname{det}(A) \neq 0$. Denote the slope of the trajectory at $x$ as

$$
\eta(x):=\frac{f_{2}(x)}{f_{1}(x)} .
$$

The vector field of (6) is partitioned into nine regions, according to the saturation function, by two vertical lines $x_{1}= \pm 1$ and two horizontal lines $x_{2}= \pm 1$ (see Fig. 1). In the central unit square, $\dot{x}=A x$.

In the region $U:=\left\{x:\left|x_{1}\right| \leq 1, x_{2} \geq 1\right\}$

$$
\begin{aligned}
& \dot{x}_{1}=-a_{11} x_{1}+a_{12} \\
& \dot{x}_{2}=-a_{21} x_{1}+a_{22} .
\end{aligned}
$$

Since $a_{11} \leq a_{12}, \dot{x}_{1} \geq 0$ in this region and the trajectories go rightward. Also note that $\dot{x}$ is independent of $x_{2}$, so for all the points on a vertical line $x_{1}=c,|c| \leq 1$ in this region $\dot{x}$ is the same. Because of this, if $x_{0} \in U$ and $\psi\left(t, x_{0}\right) \in U$ for all $t \in\left[0, t_{1}\right]$, then with $\Delta>0$

$$
\begin{array}{r}
\psi\left(t, x_{0}+\left[\begin{array}{c}
0 \\
\Delta
\end{array}\right]\right)=\psi\left(t, x_{0}\right)+\left[\begin{array}{l}
0 \\
\Delta
\end{array}\right] \\
\forall t \in\left[0, t_{1}\right] .
\end{array}
$$

We call (7) the vertical shifting property in the region $U$. Specifically, let $x_{0}=\left[\begin{array}{l}-1 \\ x_{02}\end{array}\right], x_{02} \geq 1$ be a point on the line $x_{1}=-1$, then

$$
\begin{aligned}
x_{1}(t) & =e^{-a_{11} t}(-1)+\frac{a_{12}}{a_{11}}\left(1-e^{-a_{11} t}\right) \\
& =-\left(1+\frac{a_{12}}{a_{11}}\right) e^{-a_{11} t}+\frac{a_{12}}{a_{11}} \\
x_{2}(t) & =x_{02}+\int_{0}^{t}\left(-a_{21} x_{1}(\tau)+a_{22}\right) d \tau \\
& =x_{02}+\frac{a_{21}\left(a_{12}+a_{11}\right)}{a_{11}^{2}}\left(1-e^{-a_{11} t}\right)-\frac{\operatorname{det} A}{a_{11}} t .
\end{aligned}
$$




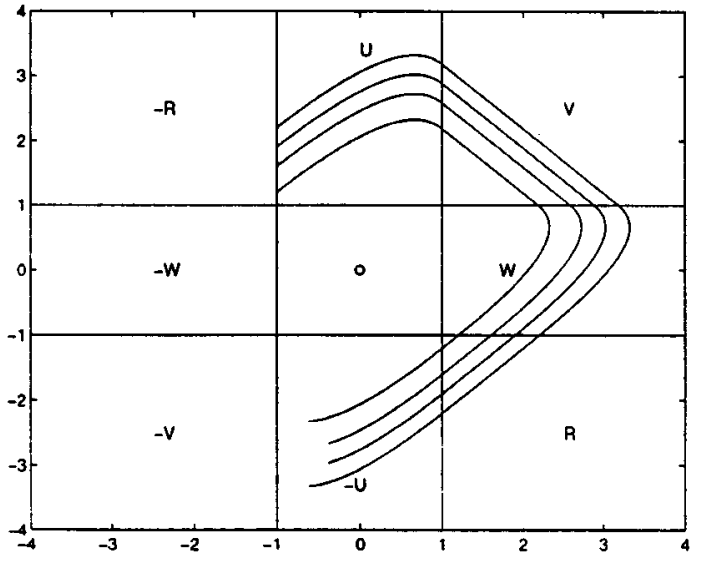

Fig. 1. The partion of the vector field.

Suppose $x(t)$ remains in the region $U$ before it intersects with the line $x_{1}=1$ at $t=T$ with $x(T)=\left[\begin{array}{c}1 \\ x_{T 2}\end{array}\right]$, then by solving (8) with $x_{1}(T)=1$ we get

$$
T=\frac{1}{a_{11}} \log \frac{a_{12}+a_{11}}{a_{12}-a_{11}}
$$

and from (9) with $t=T$ we have

$$
x_{T 2}-x_{02}=\frac{2 a_{21}}{a_{11}}-\frac{\operatorname{det} A}{a_{11}^{2}} \log \frac{a_{12}+a_{11}}{a_{12}-a_{11}} .
$$

In the above derivation, we have assumed that $a_{11}<a_{12}$. As Proposition 3.1 will show, $a_{11}=a_{12}$ automatically ensures the global asymptotic stability of the system (4) if $A$ is Hurwitz.

As expected, the increment of $x_{2}$ from $t=0$ to $t=T$ is independent of $x_{02}$. We denote this constant as

$$
h_{2}:=x_{T 2}-x_{02}=\frac{2 a_{21}}{a_{11}}-\frac{\operatorname{det} A}{a_{11}^{2}} \log \frac{a_{12}+a_{11}}{a_{12}-a_{11}} .
$$

In the region $V:=\left\{x: x_{1} \geq 1, x_{2} \geq 1\right\}$

$$
\dot{x}=\left[\begin{array}{l}
-a_{11}+a_{12} \\
-a_{21}+a_{22}
\end{array}\right]
$$

is a constant. So the slope of the trajectories is a constant. We denote this constant slope $\eta(x)$ as

$$
\alpha:=\frac{-a_{21}+a_{22}}{-a_{11}+a_{12}} .
$$

In the region $W:=\left\{x: x_{1} \geq 1,\left|x_{2}\right| \leq 1\right\}$

$$
\begin{aligned}
& \dot{x}_{1}=-a_{11}+a_{12} x_{2} \\
& \dot{x}_{2}=-a_{21}+a_{22} x_{2} .
\end{aligned}
$$

In contrast to the region $U, \dot{x}$ is independent of $x_{1}$. If $x_{0} \in W$ and $\psi\left(t, x_{0}\right) \in W$ for all $t \in\left[0, t_{1}\right]$, then with $\Delta>0$ we have

$$
\begin{array}{r}
\psi\left(t, x_{0}+\left[\begin{array}{c}
\Delta \\
0
\end{array}\right]\right)=\psi\left(t, x_{0}\right)+\left[\begin{array}{c}
\Delta \\
0
\end{array}\right] \\
\forall t \in\left[0, t_{1}\right] .
\end{array}
$$

We call (12) the horizontal shifting property in the region $W$. As Proposition 3.1 will show, if $a_{21} \leq a_{22}$, the system (4) will not be GAS. Now for the case that $a_{21}>a_{22}, \dot{x}_{2}<0$ and $\dot{x}$ points downward in this region. In this case, if a trajectory starts at a point $x_{0}=\left[\begin{array}{c}x_{01} \\ 1\end{array}\right], x_{01} \geq 1$ on the line $x_{2}=1$ and crosses the line $x_{2}=-1$ at a point $x(T)=\left[\begin{array}{c}x_{T 1} \\ -1\end{array}\right], x_{T 1} \geq 1$, then $x_{T 1}-x_{01}$ is a constant. We denote this constant as $h_{1}$. It can be verified, as with the the constant $h_{2}$, that

$$
\begin{aligned}
h_{1} & =x_{T 1}-x_{01} \\
& = \begin{cases}-\frac{2 a_{12}}{a_{22}}+\frac{\operatorname{det} A}{a_{22}^{2}} \log \frac{a_{21}+a_{22}}{a_{21}-a_{22}}, & \text { if } a_{22}>0 \\
-\frac{2 a_{11}}{a_{21}}, & \text { if } a_{22}=0 .\end{cases}
\end{aligned}
$$

In the region $R:=\left\{x: x_{1} \geq 1, x_{2} \leq-1\right\}$

$$
\dot{x}=\left[\begin{array}{l}
-a_{11}-a_{12} \\
-a_{21}-a_{22}
\end{array}\right] \text {. }
$$

We denote the constant slope $\eta(x)$ in this region as

$$
\beta:=\frac{a_{21}+a_{22}}{a_{11}+a_{12}} .
$$

The remaining four regions are symmetric to $U, V, W$, and $R$. We denote them as $-U,-V,-W,-R$.

For a general second-order nonlinear system that has a unique equilibrium point at the origin, its GAS can be proven if we can show that all its trajectories are bounded and there exists no closed trajectory. Here we have some criteria to determine the existence of closed trajectories for the system (4).

\section{Lemma 3.1:}

a) Let $Q$ be a closed bounded region that does not contain the origin. If no trajectory leaves $Q$ or no trajectory enters $Q$, then there will be a closed trajectory within $Q$.

b) Let $Q$ be a simply connected region. If $\left(\partial f_{1} / \partial x_{1}\right)+$ $\left(\partial f_{2} / \partial x_{2}\right)$ is not identically zero and does not change sign in $Q$, there will be no closed trajectory in $Q$. (Note that for $f_{1}$ and $f_{2}$ as defined by (6), $\left(\partial f_{1} / \partial x_{1}\right)+\left(\partial f_{2} / \partial x_{2}\right)$ exists inside each region of the partition of the state space Fig. 1.)

Lemma 3.1 a) is a simple application of the Poincaré-Bendixon Theorem to the system (4) and its time reversed system $\dot{x}=-A \sigma(x)$. In addition, $\mathrm{b}$ ) follows from the Bendixon Theorem. It can also be easily obtained from Green's Theorem. This theorem will be frequently applied in this paper.

If the system (4) has a closed trajectory, say, $\Gamma$, then $\Gamma$ must enclose the origin (by the index theory) since the origin is the unique equilibrium point. And $\Gamma$ must be symmetric to the origin. Since the vector field is symmetric to the origin, $-\Gamma$ is also a closed trajectory. If $\Gamma$ is not symmetric, then $\Gamma \neq-\Gamma$ and the two different trajectories will have intersections. This is impossible since no trajectories can intersect.

We next digress to address two special cases, $a_{11}=a_{12}$ and $a_{21} \leq a_{22}$.

Proposition 3.1: Assume $A$ is Hurwitz.

a) If $a_{11}=a_{12}$, then the system (6) is GAS.

b) If $a_{21} \leq a_{22}$ (which implies that $a_{11} \neq a_{12}$ ), then the system has diverging trajectories and also has a closed trajectory.

Proof:

a) We see that a) is an extension of b) in Remark 2.1 and can be proven by a method similar to [1]. First we claim that 
the vertical strip $H=\left\{x:\left|x_{1}\right| \leq 1\right\}$ is an invariant set and a global attractor. Since $A$ is Hurwitz (det $A>0$ ) and $a_{11}=a_{12}$, we must have $a_{21}>a_{22}$.

On the line $x_{1}=1, \dot{x}_{1} \leq 0$ and on $x_{1}=-1, \dot{x}_{1} \geq 0$. So no trajectory in $H$ points out of it and hence it is an invariant set. In the region $V, \dot{x}_{1}=0, \dot{x}_{2}=-a_{21}+a_{22}<$ 0 , so all the trajectories in this region will enter $W$. In $W, \dot{x}_{1} \leq 0, \dot{x}_{2}<0$, so all the trajectories will enter the central square or the region $R$. In $R, \dot{x}_{1}=-a_{11}-a_{12}<$ $0, \dot{x}_{2}=-a_{21}-a_{22}<0$, so all the trajectories will enter the region $-U$. Similar arguments apply to the regions $-V,-W,-R$. This shows that all the trajectories outside of the strip $H$ will enter it. Hence, it is a global attractor.

Next we show that all the trajectories in $H$ are bounded. Let $p_{1}=\left[\begin{array}{c}-1 \\ h\end{array}\right], h \geq 1$ be a point on the line $x_{1}=-1$. Then $\eta\left(p_{1}\right)=\beta>0$. In the region $U, \eta(x)$ depends only on $x_{1}$ and it can be easily verified that $\eta(x)$ is a decreasing function of $x_{1}$ and $\dot{x}_{1}>0$ for $x_{1} \in[-1,1)$. So if we draw a straight line $E$ with slope $\beta$ at $p_{1}$, then no trajectory in $U$ will cross $E$ upward. Symmetrically, no trajectory will cross $-E$ downward. This shows that the parallelogram enclosed by $E,-E$ and $x_{1}= \pm 1$, denoted as $P$, is also an invariant set and $\psi\left(t, x_{0}\right) \in P$ for all $t \geq 0$ as long as $x_{0} \in P$. Since for every $x_{0} \in U$ there exists such a parallelogram that encloses $x_{0}$, it follows that all the trajectories are bounded.

In $H,\left(\partial f_{1} / \partial x_{1}\right)+\left(\partial f_{2} / \partial x_{2}\right)<0\left(=-a_{11}\right.$ in $U,-U$ or $-a_{11}+a_{22}$ in the central square) so by Lemma 3.1b), there exists no closed trajectory in $H$. Since $H$ is a global attractor, all the trajectories will enter it and then converge to the origin. Thus, the system is GAS.

b) From $0 \leq a_{21} \leq a_{22}$ we have $a_{22}>0$, otherwise $\operatorname{det}(A)=0$. We also have $\alpha \geq 0,\|\dot{x}\|>0$, and $\angle \dot{x} \in$ $[0, \pi / 2]$ in the region $V$. So every trajectory starting from within this region will diverge along a straight line with slope $\alpha$ and is unbounded. Let $Q_{1}$ be the polygon with vertices $1,2, \cdots, 8$ (see Fig. 2 , where at point $2 x=$ $\left[\begin{array}{c}1 \\ a_{21} / a_{22}\end{array}\right]$ and the line from 3 to 4 has slope $\beta$ ). From 2 to $3 \dot{x}_{2}=0$ and $\dot{x}_{1}>0$. From 3 to $4, \angle \dot{x} \geq \tan ^{-1} \beta-\pi$, so the trajectories direct outward from $Q_{1}$. It is also easy to see that on other parts of the boundary of $Q_{1}$, all the trajectories remain on it or direct outward from it. Since $A$ is Hurwitz, there exists a Lyapunov level set $Q_{0}$ in the central square such that all the trajectories inside $Q_{0}$ will stay inside and converge to the origin. Let $Q=Q_{1} \backslash Q_{0}$, then no trajectory will enter $Q$, so by Lemma 3.1, there is a closed trajectory in $Q$.

Now that the two special cases are cleared, we now turn to the remaining case where $a_{11}<a_{12}$ and $a_{21}>a_{22}$. For this case, all the trajectories go clockwise, see Fig. 1 for some typical trajectories. Here we summarize the properties of the trajectories as follows.

In the region $U, \dot{x}_{1}>0$ and the trajectories go rightward. If $x_{1}<a_{22} / a_{21}, \dot{x}_{2}>0$ and if $x_{1}>a_{22} / a_{21}, \dot{x}_{2}<0$. On the line $x_{1}=a_{22} / a_{21}$, the trajectories turn from upward to downward.

In the region $W, \dot{x}_{2}<0$ and the trajectories go downward. On the line $x_{2}=a_{11} / a_{12}$, the trajectories turn from rightward to leftward.

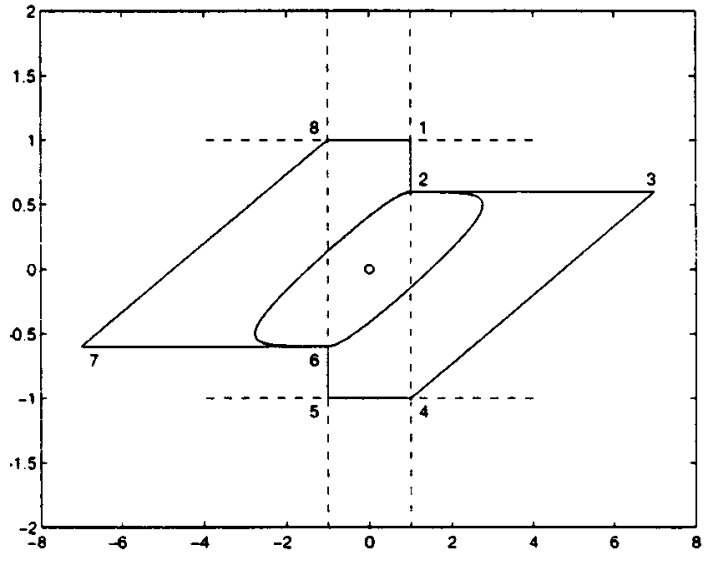

Fig. 2. Illustration for the proof of Proposition 3.1.

In the region $V$, the trajectories are straight lines that go downward-rightward; In the region $R$, the trajectories go downward-leftward.

In the central square, on the line $x_{2}=\left(a_{11} / a_{12}\right) x_{1}, \dot{x}_{1}=0$ and on the line $x_{2}=\left(a_{22} / a_{21}\right) x_{1}, \dot{x}_{2}=0$.

Finally, in this case, $h_{1}, h_{2}, \alpha$ and $\beta$ are all well defined.

\section{CONDITIONS FOR THE GlobAL BOUNDEDNESS OF THE TRAJECTORIES}

In this section, we consider the system

$$
\begin{aligned}
& \dot{x}=A \sigma(x)=\left[\begin{array}{ll}
-a_{11} & a_{12} \\
-a_{21} & a_{22}
\end{array}\right] \sigma(x) \\
& a_{11}, a_{21}, a_{12}>0, \quad a_{22} \geq 0 .
\end{aligned}
$$

Assume that $a_{11}<a_{12}$ and $a_{21}>a_{22}$ (this implies $\operatorname{det}(A) \neq$ 0 .) We do not assume that $A$ is Hurwitz in this section since the critical case where $A$ has a pair of pure imaginary eigenvalues will be useful to our study. It turns out that the system can have a bounded global attractor, even if $A$ is unstable. The global boundedness depends on $\beta / \alpha, h_{1}$ and $h_{2}$, rather than the stability of $A$.

Proposition 4.1: Assume $a_{11}<a_{12}$ and $a_{21}>a_{22}$. The system (15) has a bounded global attractor if and only if one of the following conditions is satisfied.

a) $a_{11} a_{21}>a_{12} a_{22}$.

b) $a_{11} a_{21}=a_{12} a_{22}$ and $\beta h_{1}+h_{2}<0$.

If $a_{11} a_{21}=a_{12} a_{22}$ and $\beta h_{1}+h_{2}=0$, then outside certain region, all the trajectories are closed. If $a_{11} a_{21}<a_{12} a_{22}$ ( or $a_{11} a_{21}=a_{12} a_{22}$ and $\beta h_{1}+h_{2}>0$ ), there will be unbounded trajectories and if, in addition, $A$ is Hurwitz, there exists a closed trajectory.

Proof: Under the assumption that $a_{11}<a_{12}$ and $a_{21}>$ $a_{22}$, we have $\alpha<0, \beta>0,\left|h_{1}\right|,\left|h_{2}\right|<\infty$.

Let

$$
p_{1}=\left[\begin{array}{c}
1 \\
u_{k}+1
\end{array}\right], \quad u_{k} \geq \max \left(0, \frac{\alpha}{\beta}\left(\beta h_{1}+h_{2}\right), \alpha h_{1}\right)
$$

be a point on the line $x_{1}=1$. See the point labeled 1 in Fig. 3. Let the trajectory starting from $p_{1}$ be $\psi\left(t, p_{1}\right)$. We will show later that $\psi\left(t, p_{1}\right)$ will go through regions $V, W, R$, and $-U$ consecutively (not fall into the central square before leaving 


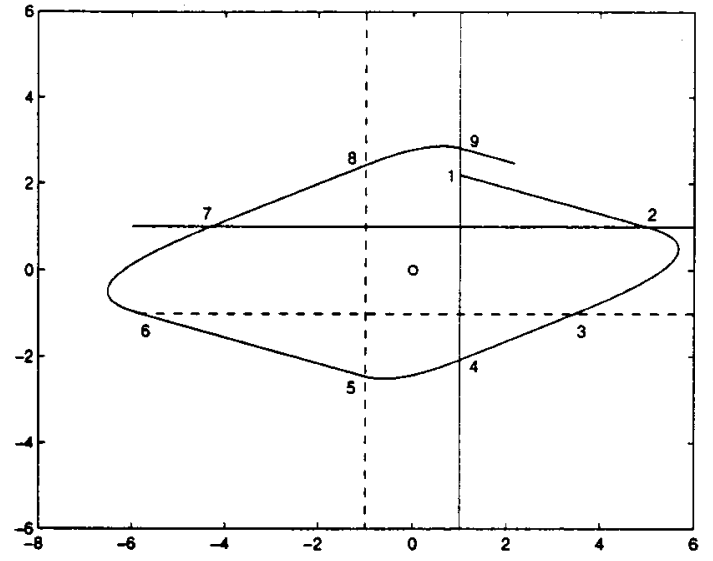

Fig. 3. Illustration for the Proof of Proposition 4.1.

$-U)$. Let the intersections of $\psi\left(t, p_{1}\right)$ with the lines $x_{2}=$ $1, x_{2}=-1, x_{1}=1, x_{1}=-1$ be

$$
\left[\begin{array}{c}
1+v_{k} \\
1
\end{array}\right],\left[\begin{array}{c}
1+w_{k} \\
-1
\end{array}\right],\left[\begin{array}{c}
1 \\
-1-r_{k}
\end{array}\right] \text { and }\left[\begin{array}{c}
-1 \\
-1-u_{k+1}
\end{array}\right]
$$

which correspond to the points 2, 3, 4, 5 in Fig. 3. Then

$$
\begin{aligned}
& v_{k}=-\frac{1}{\alpha} u_{k} \\
& w_{k}=v_{k}+h_{1}=-\frac{1}{\alpha} u_{k}+h_{1} \\
& r_{k}=\beta w_{k}=-\frac{\beta}{\alpha} u_{k}+\beta h_{1}
\end{aligned}
$$

and $u_{k+1}=r_{k}+h_{2}$, i.e.,

$$
u_{k+1}=-\frac{\beta}{\alpha} u_{k}+\beta h_{1}+h_{2} \text {. }
$$

The requirement that the trajectory does not enter the central square is equivalent to $v_{k}, w_{k}, r_{k}, u_{k+1} \geq 0$. This can be guaranteed by $u_{k} \geq \max \left(0,(\alpha / \beta)\left(\beta h_{1}+h_{2}\right), \alpha h_{1}\right)$. If we also have $u_{k+1} \geq \max \left(0,(\alpha / \beta)\left(\beta h_{1}+h_{2}\right), \alpha h_{1}\right)$, then we can continue with the above process symmetrically to get an intersection with the line $x_{1}=1,\left[\begin{array}{c}1 \\ 1+u_{k+2}\end{array}\right]$ (point 9 in Fig. 3) where

$$
u_{k+2}=-\frac{\beta}{\alpha} u_{k+1}+\beta h_{1}+h_{2}
$$

and so on. Equation (16) defines a first-order linear time invariant discrete-time system.

Case $1\left(a_{11} a_{21}<a_{12} a_{22}\right)$ : This inequality is equivalent to $-(\beta / \alpha)>1$. So in this case, the discrete-time system (16) is unstable. If $u_{k}>$ $\max \left(0,(\alpha /(\alpha+\beta))\left(\beta h_{1}+h_{2}\right),(\alpha / \beta)\left(\beta h_{1}+h_{2}\right), \alpha h_{1}\right)$, then $u_{k+1}>u_{k}, u_{k+2}>u_{k+1}, \cdots$ will be an exponentially increasing sequence and the trajectory starting from $\left[\begin{array}{c}1 \\ u_{k}+1\end{array}\right]$ will be unbounded.

Let $p_{1}=\left[\begin{array}{c}1 \\ 1+u_{1}\end{array}\right]$ where $u_{1}>$ $\max \left(0,(\alpha /(\alpha+\beta))\left(\beta h_{1}+h_{2}\right),(\alpha / \beta)\left(\beta h_{1}+h_{2}\right), \alpha h_{1}\right)$ (see point 1 in Fig. 4). Then by the foregoing argument, $\psi\left(t, p_{1}\right)$ will return to the line $x_{1}=1$ at a point above $p_{1}$ (see 2 in Fig. 4). By connecting 1 and 2, we get a closed curve. Let the region enclosed by this closed curve be $Q_{1}$. From 1 to $2, \dot{x}$ is a constant and $\angle \dot{x} \in(-(\pi / 2), 0)$ since $\alpha<0$. So $\dot{x}$ directs

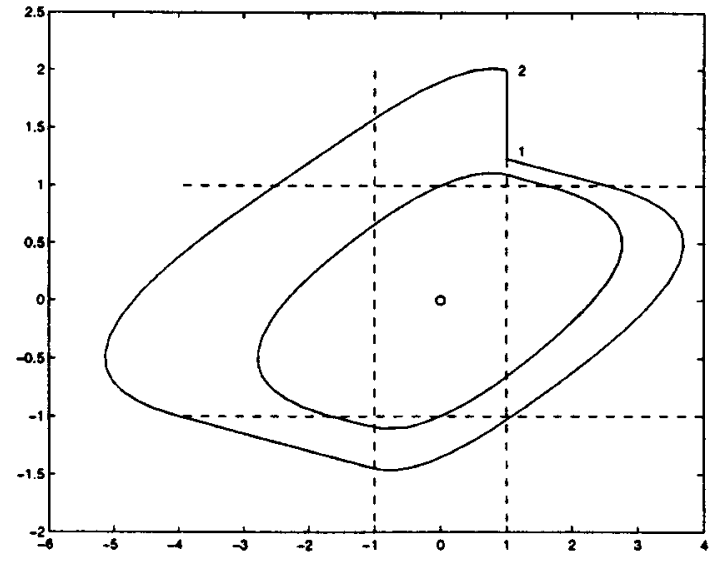

Fig. 4. Illustration for the Proof of Proposition 4.1: Case 1.

outward from $Q_{1}$. If $A$ is Hurwitz, there will be a Lyapunov level set $Q_{0}$ in the central square which is invariant. Let $Q=Q_{1} \backslash Q_{0}$, then no trajectory will enter $Q$ and, by Lemma 3.1, there is a closed trajectory in $Q$ as illustrated in Fig. 4.

Case $2\left(a_{11} a_{21}>a_{12} a_{22}\right)$ : This inequality is equivalent to $-(\beta / \alpha)<1$. Since $-(\beta / \alpha)>0$, in this case the discrete-time system (16) is stable. Let $u^{*}$ be chosen such that

$$
\begin{aligned}
& \min \left(-\frac{\beta}{\alpha} u^{*}+\beta h_{1}+h_{2}, u^{*}\right) \\
& >\max \left(0, \frac{\alpha}{\beta}\left(\beta h_{1}+h_{2}\right), \alpha h_{1}\right)
\end{aligned}
$$

and

$$
\left(\left(\frac{\beta}{\alpha}\right)^{2}-1\right) u^{*}+\left(1-\frac{\beta}{\alpha}\right)\left(\beta h_{1}+h_{2}\right)<-1
$$

then with $u_{k} \geq u^{*}$, the trajectory $\psi\left(t,\left[\begin{array}{c}1 \\ 1\end{array}\right]\right)$ does not fall into the central square before it returns to the line $x_{1}=1$ (This is guaranteed by (17). Moreover, because of (18), we have

$$
\begin{aligned}
u_{k+2}-u_{k} & =\left(\left(\frac{\beta}{\alpha}\right)^{2}-1\right) u_{k}+\left(1-\frac{\beta}{\alpha}\right)\left(\beta h_{1}+h_{2}\right) \\
& \leq\left(\left(\frac{\beta}{\alpha}\right)^{2}-1\right) u^{*}+\left(1-\frac{\beta}{\alpha}\right)\left(\beta h_{1}+h_{2}\right) \\
& <-1
\end{aligned}
$$

Let $p_{1}=\left[\begin{array}{c}1 \\ 1+u^{*}\end{array}\right]$ (see point 1 in Fig. 5). Then by the foregoing argument, $\psi\left(t, p_{1}\right)$ will return to the line $x_{1}=1$ at a point 2 between $p_{1}$ and [ $\left[\begin{array}{l}1 \\ 1\end{array}\right]$. By connecting 1 and 2, we get a closed curve. Denote the region enclosed by this closed curve as $Q_{1}$. Since on the line between 1 and $2 \dot{x}$ directs inward of $Q_{1}$. Thus, $\psi\left(t, x_{0}\right)$ will stay in $Q_{1}$ as long as $x_{0} \in Q_{1}$. Therefore, $Q_{1}$ is an invariant set.

Let $x_{0}$ be any point outside of $Q_{1}$, then $\psi\left(t, x_{0}\right)$ goes clockwise and will intersect with the line $x_{1}=1$ above 2 , say at $p=\left[\begin{array}{c}1 \\ 1+u_{0}\end{array}\right]$. If $u_{0}<u^{*}$, i.e., $p$ is between 1 and 2 , then $\psi\left(t, x_{0}\right)$ will enter $Q_{1}$ afterward and stay there. If $u_{0} \geq u^{*}$, then by (19), we have $u_{2}<u_{0}-1, u_{4}<u_{2}-1, \cdots$ until $u_{k}<u^{*}$ for some finite $k$. This implies $\psi\left(t_{1}, x_{0}\right) \in Q_{1}$ for some $t_{1}>0$. Therefore, $Q_{1}$ is a global attractor. 


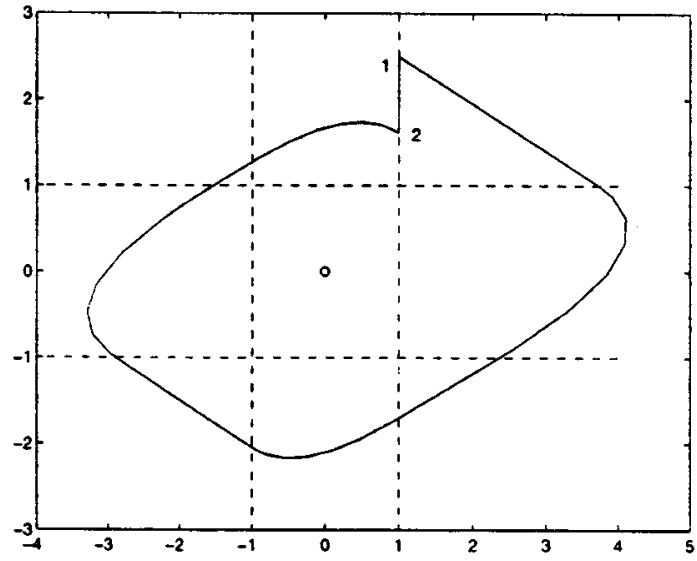

Fig. 5. Illustration for the Proof of Proposition 4.1: Case 2.

Case $3 a_{11} a_{21}=a_{12} a_{22}:$ In this case, $-(\beta / \alpha)=1$ and

$$
u_{k+2}-u_{k}=2\left(\beta h_{1}+h_{2}\right) .
$$

Suppose $\beta h_{1}+h_{2}<0$, the sequence $u_{k}, u_{k+2}, \cdots$ will decrease steadily before the trajectory touches the central square, similar to Case 2, there exists a global attractor.

Suppose $\beta h_{1}+h_{2}>0$, the sequence will increase steadily and the trajectory will go unbounded. Also, similarly to Case 2, there exists a closed trajectory.

Suppose $\beta h_{1}+h_{2}=0$, then if $u_{k} \geq \max \left(0,(\alpha / \beta)\left(\beta h_{1}+h_{2}\right), \alpha h_{1}\right)$, we will have $u_{k}=u_{k+1}=\cdots=u_{k+N}$ for all $N$. So $\left[\begin{array}{c}1 \\ 1+u_{k}\end{array}\right]$ is on a closed trajectory. Let $Q_{1}$ be the region enclosed by the closed trajectory passing through $\left[\begin{array}{c}1 \\ 1+u_{k}\end{array}\right]$, then all the trajectories outside of $Q_{1}$ are closed.

To demonstrate Proposition 4.1, consider the system with $A=\left[\begin{array}{ll}-1 & 2 \\ -5 & 2\end{array}\right]$. Clearly, $A$ is exponentially unstable, but $a_{11} a_{21}>$ $a_{12} a_{22}$. So the system has a global attractor (see Fig. 6).

An interesting case is that $a_{11}=a_{22}$ and $a_{21}=a_{12}$. In this case, $A$ has a pair of pure imaginary eigenvalues. For the linear system $\dot{x}=A x$ every point in the plane is on a closed trajectory. This is also true for the saturated system

$$
\dot{x}=A \sigma(x)=\left[\begin{array}{ll}
-a_{11} & a_{12} \\
-a_{12} & a_{11}
\end{array}\right] \sigma(x), \quad a_{12}>a_{11}>0 .
$$

Denote the trajectory of (20) as $\psi_{1}\left(t, x_{0}\right)$.

Proposition 4.2: All the trajectories of (20) are closed. Each trajectory is symmetric with respect to the line $x_{1}=x_{2}$ and the line $x_{1}=-x_{2}$.

Proof: For this system, $a_{11} a_{21}=a_{12} a_{22}$ and it can also be verified that $\beta h_{1}+h_{2}=0$. By Proposition 4.1, $\psi_{1}\left(t, x_{0}\right)$ is bounded for every $x_{0}$. On the other hand, since $A$ has a pair of pure imaginary eigenvalues, there are closed trajectories in any neighborhood of the origin. Thus, any $x_{0}$ is outside of a closed trajectory. Therefore, $\psi_{1}\left(t, x_{0}\right)$ will be a closed curve or go to a closed curve. Since $\psi_{1}\left(t, x_{0}\right)$ goes clockwise, it will intersect the line $x_{1}=x_{2}$ somewhere, say, at $\left[\begin{array}{r}r \\ r\end{array}\right]$. So, for simplicity, we can assume that $x_{0}=\left[\begin{array}{r}r \\ r\end{array}\right]$ for some $r>0$. To show $\psi_{1}\left(t, x_{0}\right)$ is a closed trajectory and is symmetric to the line $x_{1}=x_{2}$, it suffices to show that $\psi_{1}\left(t, x_{0}\right)=J \psi_{1}\left(-t, x_{0}\right)$ where $J=\left[\begin{array}{ll}0 & 1 \\ 1 & 0\end{array}\right]$.

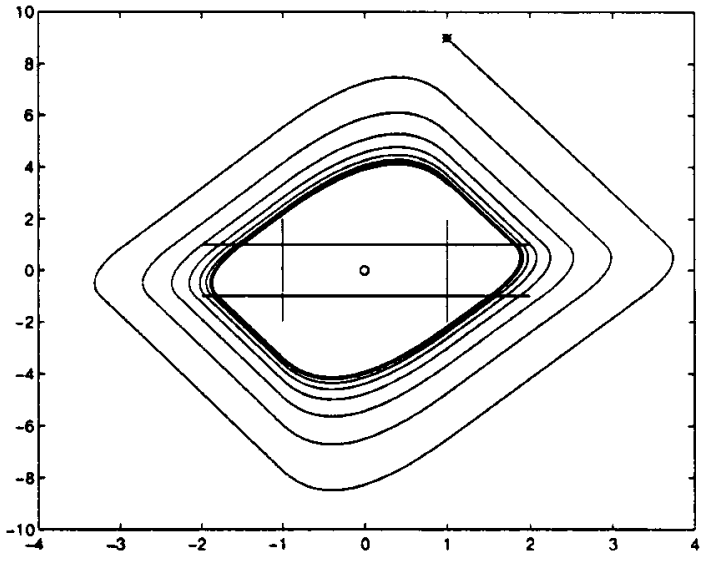

Fig. 6. A global attractor.

Consider the time-reversed system of (20),

$$
\dot{z}=-A \sigma(z) \text {. }
$$

Denote its trajectory as $\phi\left(t, x_{0}\right)$. Then $\psi_{1}\left(-t, x_{0}\right)=\phi\left(t, x_{0}\right)$. From (21), we have

$$
J \dot{z}=-J A \sigma(z)=-J A J \sigma(J z)=A \sigma(J z)
$$

thus, $J \phi\left(t, x_{0}\right)=\psi_{1}\left(t, J x_{0}\right)$. Since $J x_{0}=x_{0}$, it follows that $\psi_{1}\left(t, x_{0}\right)=J \psi_{1}\left(-t, x_{0}\right)$.

To show that the trajectory is symmetric with respect to $x_{1}=$ $-x_{2}$, we write (20) as

$$
\begin{aligned}
{\left[\begin{array}{r}
\dot{x}_{1} \\
-\dot{x}_{2}
\end{array}\right] } & =\left[\begin{array}{rr}
-a_{11} & -a_{12} \\
a_{12} & a_{11}
\end{array}\right]\left[\begin{array}{c}
\sigma\left(x_{1}\right) \\
\sigma\left(-x_{2}\right)
\end{array}\right] \\
& =A^{T}\left[\begin{array}{c}
\sigma\left(x_{1}\right) \\
\sigma\left(-x_{2}\right)
\end{array}\right] .
\end{aligned}
$$

We also have $-J A^{T} J=A^{T}$. Following the same procedure as above by considering the state $\left[\begin{array}{c}x_{1} \\ -x_{2}\end{array}\right]$ instead, we can show that all the trajectories are symmetric with respect to the line $x_{1}=-x_{2}$.

The system (20) is not GAS but is useful for us to develop the condition for global asymptotic stability. We will establish the main result of the paper by comparing the trajectory of a general system with that of (20).

\section{Proof of Theorem 2.1}

In view of Remark 2.1 and Proposition 3.1, we only need to consider the following system:

$$
\begin{array}{r}
\dot{x}=A \sigma(x)=\left[\begin{array}{ll}
-a_{11} & a_{12} \\
-a_{21} & a_{22}
\end{array}\right] \sigma(x) \\
a_{11}, a_{12}, a_{21}>0, a_{22} \geq 0
\end{array}
$$

with $a_{12}>a_{11}, a_{21}>a_{22}$.

Proposition 5.1: Assume that $a_{12}>a_{11}$ and $A$ is Hurwitz, the system (22) is GAS if and only if $a_{11} a_{21} \geq a_{12} a_{22}$.

This proposition can be established as follows. First note that $a_{12}>a_{11}$ and $a_{11} a_{21} \geq a_{12} a_{22}$ imply $a_{21}>a_{22}$. If $a_{11} a_{21}=$ $a_{12} a_{22}$ and $A$ is Hurwitz, then it can be verified that $\beta h_{1}+$ $h_{2}<0$. Hence, this proposition shows that the stability of $A$ 
along with the global boundedness condition in Proposition 4.1 guarantees the system to be GAS.

If $a_{11} a_{21}<a_{12} a_{22}$, then by Propositions 3.1 and 4.1 , the system is not GAS whether $a_{21}>a_{22}$ or not. So the necessity of the condition is obvious. What remains is to show the sufficiency of the condition. Now that the global boundedness of the trajectories is guaranteed, the only thing needed to be shown is that the system has no closed trajectory.

Since all the trajectories are kept unchanged when the vector field is multiplied by a positive constant, we assume that $a_{11}=$ 1 in the sequel for simplicity. Now we have

$$
A=\left[\begin{array}{cc}
-1 & a_{12} \\
-a_{21} & a_{22}
\end{array}\right] \text {. }
$$

We first deal with the case where $a_{22}=0$.

Lemma 5.1: Assume $a_{12}>a_{11}$ and $A$ is Hurwitz. If $a_{22}=$ 0 , then (22) is GAS.

Proof: See the Appendix.

In what follows, we consider the case that $a_{22}>0$. Let $k=$ $a_{21} / a_{12} a_{22}$, then we can assume that $A$ takes the form

$$
A=\left[\begin{array}{cc}
-1 & a_{12} \\
-k a_{12} a_{22} & a_{22}
\end{array}\right], \quad k>0, a_{22}>0 .
$$

The assumption in Proposition 5.1 that $a_{11}<a_{12}$ and $A$ is Hurwitz translates to

$$
a_{12}>1, \quad a_{22}<1, \quad k a_{12}^{2}>1
$$

and the condition $a_{11} a_{21} \geq a_{12} a_{22}$ is equivalent to

$$
k \geq 1
$$

Therefore, we can establish Proposition 5.1 by showing that the system

$$
\begin{array}{r}
\dot{x}=A \sigma(x)=\left[\begin{array}{cc}
-1 & a_{12} \\
-k a_{12} a_{22} & a_{22}
\end{array}\right] \sigma(x) \\
a_{12}>1, \quad k \geq 1, \quad 0<a_{22}<1
\end{array}
$$

is GAS. The proof will be carried out by evolving $A$ from the simplest form where $a_{22}=1, k=1$ to the case $a_{22}=1, k \geq 1$ and finally to the general case $0<a_{22}<1, k \geq 1$. When $a_{22}=1$, the system is surely not GAS because $A$ is not Hurwitz, but the trajectories in this case will be used as a reference to show the convergence of the trajectories when $a_{22}$ is decreased.

To proceed, we need a technical lemma. Recall (13)

$$
h_{1}= \begin{cases}-\frac{2 a_{12}}{a_{22}}+\frac{\operatorname{det} A}{a_{22}^{2}} \log \frac{a_{21}+a_{22}}{a_{21}-a_{22}}, & \text { if } a_{22}>0 \\ -\frac{2 a_{11}}{a_{21}}, & \text { if } a_{22}=0 .\end{cases}
$$

Now we have $a_{22}>0$ and $a_{21}=k a_{12} a_{22}$, so

$$
h_{1}=\frac{1}{a_{22}}\left(-2 a_{12}+\left(k a_{12}^{2}-1\right) \log \frac{k a_{12}+1}{k a_{12}-1}\right) .
$$

Lemma 5.2: If $a_{22}>0, a_{12}>1$ and $k \geq 1$, then $h_{1}<0$. Proof: See the Appendix.
Now we consider the case where $a_{22}=1$ and $k \geq 1$,

$$
\dot{x}=\left[\begin{array}{cc}
-1 & a_{12} \\
-k a_{12} & 1
\end{array}\right] \sigma(x), \quad a_{12}>1, \quad k \geq 1 .
$$

Given an initial point $x_{0}$, denote the trajectory of (25) as $\psi_{2}\left(t, x_{0}\right)$ and as a comparison, denote the trajectory of

$$
\dot{x}=\left[\begin{array}{cc}
-1 & a_{12} \\
-a_{12} & 1
\end{array}\right] \sigma(x)
$$

as $\psi_{1}\left(t, x_{0}\right)$. Then $\psi_{1}\left(t, x_{0}\right)$ is closed for every $x_{0}$ by Proposition 4.2.

In the following, we present three lemmas about the intersections of $\psi_{2}\left(t, x_{0}\right)$ with some straight lines.

Lemma 5.3: Assume $k>1$.

a) Let $x_{0}=\left[\begin{array}{c}x_{01} \\ 1\end{array}\right]$. If $x_{01} \in\left(1 / a_{12}, 1\right]$, then $\psi_{2}\left(t, x_{0}\right)$ (see the dashed curve in Fig. 7) may intersect with the line $x_{1}=1$. Let the first intersection be $x_{c}=\left[\begin{array}{c}1 \\ x_{c 2}\end{array}\right]$, then $x_{c 2}<x_{01}$, i.e,

$$
\left\|x_{0}-\left[\begin{array}{l}
1 \\
1
\end{array}\right]\right\|<\left\|x_{c}-\left[\begin{array}{l}
1 \\
1
\end{array}\right]\right\| .
$$

If $x_{01} \in\left[1 / k a_{12}, 1 / a_{12}\right], \psi_{2}\left(t, x_{0}\right)$ will go downwardrightward at first, but will not intersect with the line $x_{1}=$ 1 before it turns leftward (see the dotted curve in Fig. 7).

b) Let $x_{0}=\left[\begin{array}{c}1 \\ x_{02}\end{array}\right], x_{02}<1 / a_{12}$. Then $\psi_{2}\left(t, x_{0}\right)$ goes downward-leftward (see the dash-dotted curve in Fig. 7). Let the first intersection of $\psi_{2}\left(t, x_{0}\right)$ with the line $x_{2}=$ -1 be $x_{c}=\left[\begin{array}{c}x_{c 1} \\ -1\end{array}\right]$, then $x_{02}>-x_{c 1}$, i.e.,

$$
\left\|x_{0}-\left[\begin{array}{r}
1 \\
-1
\end{array}\right]\right\|>\left\|x_{c}-\left[\begin{array}{r}
1 \\
-1
\end{array}\right]\right\| \text {. }
$$

As a comparison, two $\psi_{1}\left(t, x_{0}\right)$ are also shown in Fig. 7 (see the solid curves). In Fig. 7, $x_{0}$ 's are marked with *.

Proof: See the Appendix.

Lemma 5.4: Given $\gamma \geq 0$, let $x_{0}=\left[\begin{array}{c}1+\gamma \\ s_{1}\end{array}\right], s_{1} \in\left(1 / a_{12}, 1\right]$ be a point on the line $x_{1}=1+\gamma$. Then $\psi_{2}\left(t, x_{0}\right)$ will go downward-rightward at first, then turn leftward and return to the line $x_{1}=1+\gamma$. Let the intersection be $x_{c}=\left[\begin{array}{c}1+\gamma \\ s_{2}\end{array}\right]$, then $s_{1}+s_{2}>0$.

Proof: See the Appendix.

Lemma 5.5: Let $x_{0}=\left[\begin{array}{c}x_{01} \\ 1\end{array}\right], x_{01}>1 / k a_{12}$ be a point on the line $x_{2}=1$. Then $\psi_{2}\left(t, x_{0}\right)$ goes downward-rightward at first and turns leftward. Suppose $\psi_{2}\left(t, x_{0}\right)$ has an intersection with the line $x_{2}=-1$ at $x_{c}=\left[\begin{array}{c}x_{c 1} \\ -1\end{array}\right]$, then $x_{c 1}<x_{01}$ (see Fig. 8).

Proof: See the Appendix

The following two lemmas give a complete characterization of the trajectories of the system (25).

Lemma 5.6: Assume $k>1$. Let $x^{*}=\left[\begin{array}{c}1 / k a_{12} \\ 1\end{array}\right]$. Then, $\psi_{2}\left(t, x^{*}\right)$ is a closed curve that lies within the central square. Denote the region enclosed by $\psi_{2}\left(t, x^{*}\right)$ as $S_{0}$, then every point inside $S_{0}$ is on a closed trajectory. And outside $S_{0}$, any trajectory will converge to $\psi_{2}\left(t, x^{*}\right)$ (see Fig. 9).

Proof: See the Appendix.

Lemma 5.7: Assume $k>1$. Let $x_{0}=\left[\begin{array}{c}x_{01} \\ 1\end{array}\right], x_{01}<1 / k a_{12}$ be a point on the line $x_{2}=1$, then $\psi_{2}\left(t, x_{0}\right)$ goes upward at first and will return to the line $x_{2}=1$. Suppose $\psi_{2}\left(t, x_{0}\right)$ intersects 


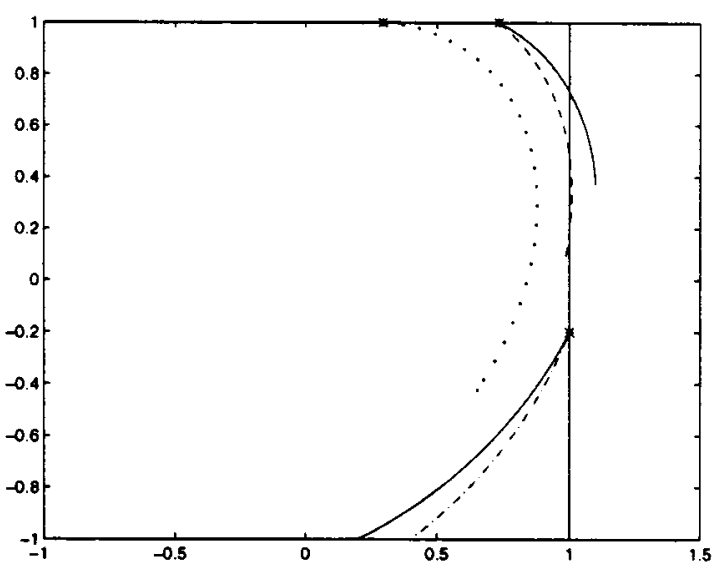

Fig. 7. Illustration for Lemma 5.3.

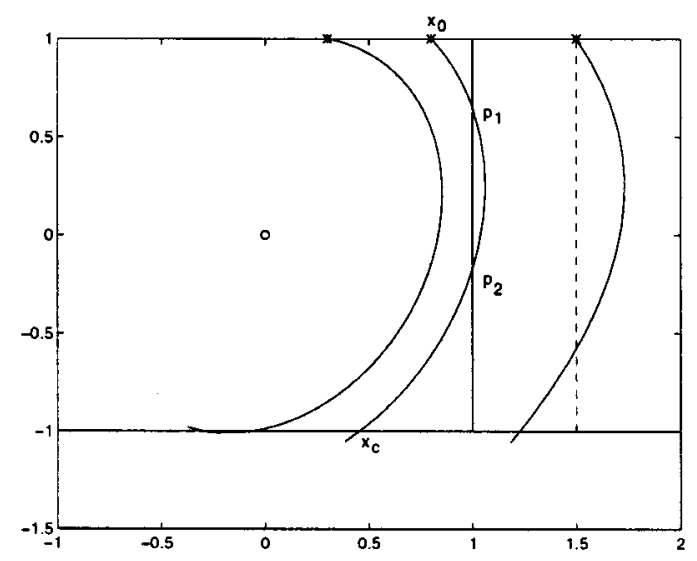

Fig. 8. Illustration for Lemma 5.5.

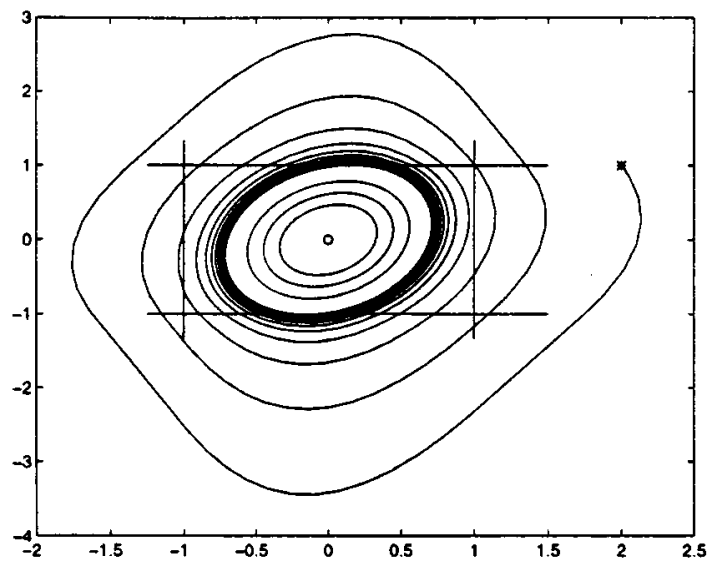

Fig. 9. Illustration for Lemma 5.6.

with the line $x_{2}=-1$. Let $x_{c}=\left[\begin{array}{c}x_{c 1} \\ -1\end{array}\right]$ be the first intersection, then $-\left(1 / k a_{12}\right)<x_{c 1}<-x_{01}$.

Proof: See the Appendix.

Lemmas 5.6 and 5.7 give us a clear picture of the trajectories of (25), where $a_{22}=1, k>1$. Lemma 5.7 shows that if $x_{0}$ is outside of $S_{0}$, a trajectory $\psi_{2}\left(t, x_{0}\right)$ will move closer and closer to $S_{0}$ as it reaches the lines $x_{2}= \pm 1$. Next we will show that as $a_{22}$ is decreased, a trajectory $\psi\left(t, x_{0}\right)$ of (24) will move even closer to $S_{0}$, as compared with $\psi_{2}\left(t, x_{0}\right)$. This will lead to our final result about the global asymptotic stability of the system
(24) and hence the proof of Proposition 5.1. Rewrite (24) as follows:

$$
\begin{aligned}
& \dot{x}_{1}=-\sigma\left(x_{1}\right)+a_{12} \sigma\left(x_{2}\right) \\
& \dot{x}_{2}=a_{22}\left(-k a_{12} \sigma\left(x_{1}\right)+\sigma\left(x_{2}\right)\right)
\end{aligned}
$$

where $a_{12}>1, k \geq 1$ and $0<a_{22}<1$. We will consider the perturbation of the trajectories as $a_{22}$ is varied, so denote the trajectory of (27) as $\psi\left(t, x_{0}, a_{22}\right)$ and the slope of a trajectory at $x$ be $\eta\left(x, a_{22}\right)$. As compared with (25), $\dot{x}_{1}$ is the same but $\dot{x}_{2}$ is multiplied with a scalar $a_{22}$. Because of this, the trajectories of (27) exhibit some interesting properties.

\section{Fact 5.1:}

a) Let $x_{0}=\left[\begin{array}{l}x_{01} \\ x_{02}\end{array}\right], x_{02} \geq 1$ be a point above the line $x_{2}=1$, then for all $a_{22}>0$

$\left[\begin{array}{ll}0 & 1\end{array}\right] \psi\left(t, x_{0}, a_{22}\right)-x_{02}=a_{22}\left(\left[\begin{array}{ll}0 & 1\end{array}\right] \psi_{2}\left(t, x_{0}\right)-x_{02}\right)$

$$
\left[\begin{array}{ll}
1 & 0
\end{array}\right] \psi\left(t, x_{0}, a_{22}\right)=\left[\begin{array}{ll}
1 & 0
\end{array}\right] \psi_{2}\left(t, x_{0}\right)
$$

as long as $\psi\left(t, x_{0}, a_{22}\right)$ stays above the line $x_{2}=1$.

b) Let $x_{0}=\left[\begin{array}{l}x_{01} \\ x_{02}\end{array}\right], x_{01} \geq 1$ be a point to the right of the line $x_{1}=1$, then for all $a_{22}>0$

$$
\begin{aligned}
& {\left[\begin{array}{ll}
1 & 0
\end{array}\right] \psi\left(t, x_{0}, a_{22}\right)-x_{01}} \\
& \quad=\frac{1}{a_{22}}\left(\left[\begin{array}{ll}
1 & 0
\end{array}\right] \psi_{2}\left(a_{22} t, x_{0}\right)-x_{01}\right)
\end{aligned}
$$

$$
\left[\begin{array}{ll}
0 & 1
\end{array}\right] \psi\left(t, x_{0}, a_{22}\right)=\left[\begin{array}{ll}
0 & 1
\end{array}\right] \psi_{2}\left(a_{22} t, x_{0}\right)
$$

as long as $\psi\left(t, x_{0}, a_{22}\right)$ stays to the right of $x_{1}=1$.

See Fig. 10 for an illustration, where the solid curves are $\psi_{2}\left(t, x_{0}\right)$ and $\psi_{2}\left(a_{22} t, x_{0}\right)$, and the dashed curves are $\psi\left(t, x_{0}, a_{22}\right), a_{22}<1$.

Fact 5.1a) implies that $\psi\left(t, x_{0}, a_{22}\right)$ and $\psi_{2}\left(t, x_{0}\right)$ are on the same vertical line but the distance from $\psi\left(t, x_{0}, a_{22}\right)$ to the line $x_{2}=x_{02}$ is $a_{22}$ times that from $\psi_{2}\left(t, x_{0}\right)$ to $x_{2}=x_{02}$. In particular, $\psi_{2}\left(t, x_{0}\right)$ and $\psi\left(t, x_{0}, a_{22}\right)$ return to the line $x_{2}=x_{02}$ at the same time and the same point. This simply follows from the fact that $\dot{x}_{2}$ of (27) is $a_{22}$ times that of (25) and that $\dot{x}$ is independent of $x_{2}$ above the line $x_{2}=1$. It can also be directly verified from the expression of $\psi\left(t, x_{0}, a_{22}\right)$ and $\psi_{2}\left(t, x_{0}\right)$.

Fact 5.1b) implies that $\psi\left(t, x_{0}, a_{22}\right)$ and $\psi_{2}\left(a_{22} t, x_{0}\right)$ are on the same horizontal line but the distance from $\psi\left(t, x_{0}, a_{22}\right)$ to the line $x_{1}=x_{01}$ is $1 / a_{22}$ times that from $\psi_{2}\left(a_{22} t, x_{0}\right)$ to $x_{1}=x_{01}$. In particular, $\psi_{2}\left(a_{22} t, x_{0}\right)$ and $\psi\left(t, x_{0}, a_{22}\right)$ return to the line $x_{1}=x_{01}$ at the same time and the same point. This also follows from the fact that $\dot{x}_{2}$ is scaled by $a_{22}$. If we scale the vector field to the right of the line $x_{1}=1$ by $1 / a_{22}$, then $\dot{x}_{2}$ is the same as that of (25) but $\dot{x}_{1}$ is amplified by $1 / a_{22}$. Note that the scaling of the vector field results in the time scaling of $\psi_{2}\left(a_{22} t, x_{0}\right)$.

With Fact 5.1, we are ready to present a final lemma that leads to the proof of Proposition 5.1. 


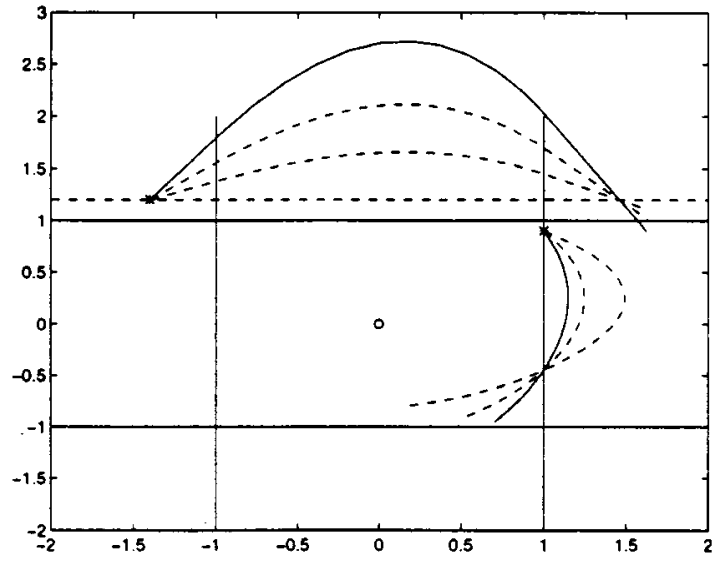

Fig. 10. Illustration for Fact 5.1.

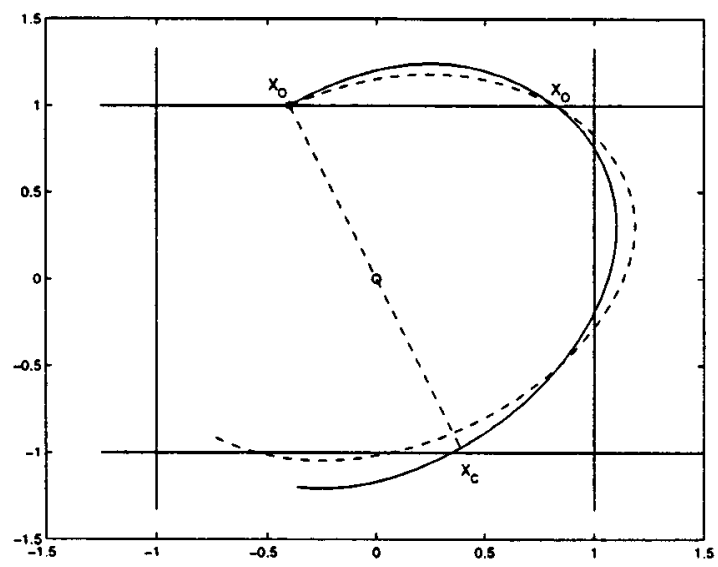

Fig. 11. Illustration for the Proof of Proposition 5.1.

Lemma 5.8: Let $x_{0}=\left[\begin{array}{l}x_{01} \\ x_{02}\end{array}\right], x_{02} \in(0,1], x_{01} \geq$ $x_{02} / k a_{12}$. Suppose $\psi\left(t, x_{0}, a_{22}\right)$ intersects with the line $x_{2}=-x_{02}$ at a point $x_{c}=\left[\begin{array}{c}x_{01} \\ -x_{02}\end{array}\right]$ and $x_{c 1}<x_{01}$, then if $\delta \in\left(0, a_{22}\right)$ is sufficiently small, $\psi\left(t, x_{0}, a_{22}-\delta\right)$ will intersect with $\psi\left(t, x_{0}, a_{22}\right)$ at a point to the right of $x_{0}$. If it also intersects with the line $x_{2}=-x_{02}$, the intersection will be to the left of $x_{c}$.

For an illustration of Lemma 5.8, see Figs. 16-18 where the solid curves are $\psi\left(t, x_{0}, a_{22}\right)$ and the dashed curves are $\psi\left(t, x_{0}, a_{22}-\delta\right)$.

Proof: See the Appendix.

Proof of Proposition 5.1: The necessity of the condition simply follows from Propositions 3.1 and 4.1. With Lemma 5.1, it remains to be shown that the system (27) or (24) is GAS. We will first show that any point on the line $x_{2}=1$ is not on a closed trajectory. We can restrict our attention to the points to the left of $\left[\begin{array}{c}1 / k a_{12} \\ 1\end{array}\right.$, since for the points to its right, they can be traced back to the left as the trajectories go rightward above the line $x_{2}=1$. Let $x_{0}=\left[{ }^{x_{01}}{ }_{1}\right], x_{01}<1 / k a_{12}$, then $\psi_{2}\left(t, x_{0}\right)$ of the system (25) (see the solid curve in Fig. 11) will return to the line $x_{2}=1$ at a point $x_{0}^{\prime}$. From Fact 5.1, $\psi\left(t, x_{0}, a_{22}\right)$ will also return to $x_{0}^{\prime}$ for all $a_{22}>0$ (see the dashed curve in Fig. 11).

We have shown in Lemma 5.7 that for any $x_{0}$ to the left of $\left[\begin{array}{c}1 / k a_{12} \\ 1\end{array}\right]$, if $\psi_{2}\left(t, x_{0}\right)$ reaches the line $x_{2}=-1$ at some point

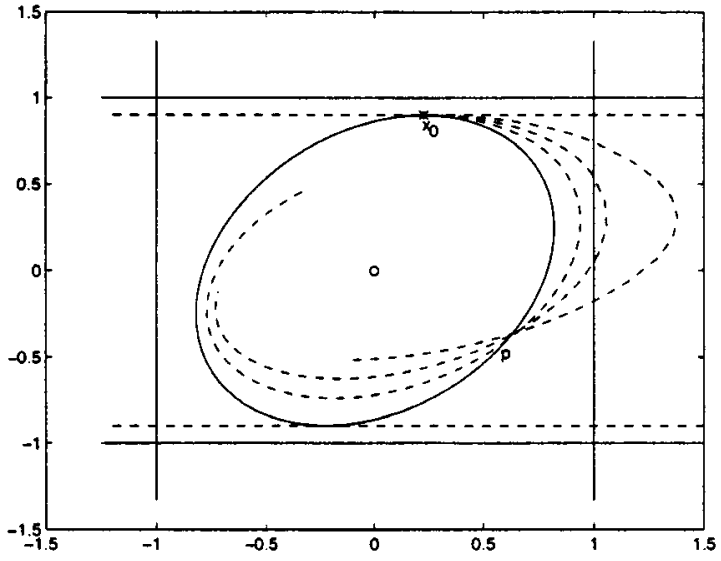

Fig. 12. Illustration for the Proof of Proposition 5.1.

$x_{c}=\left[\begin{array}{c}x_{c 1} \\ -1\end{array}\right]$, then $x_{c 1}<-x_{01}$, i.e., $x_{c}$ is to the left of $-x_{0}$. By Lemma 5.5, $x_{c}$ is also to the left of $x_{0}^{\prime}$. From Lemma 5.8, we know that as $a_{22}$ is decreased from 1 , the intersection of $\psi\left(t, x_{0}^{\prime}, a_{22}\right)$ and $x_{2}=-1$ will move leftward and, hence, remain to the left of $-x_{0}$ and $x_{0}^{\prime}$. Note that $x_{0}^{\prime}$ is on $\psi\left(t, x_{0}, a_{22}\right)$, so $\psi\left(t, x_{0}^{\prime}, a_{22}\right)$ overlaps with $\psi\left(t, x_{0}, a_{22}\right)$. Therefore, $x_{0}$ is not on a closed trajectory (note that a closed trajectory must be symmetric).

Next we exclude the possibility of the existence of a closed trajectory that does not intersect with $x_{2}=1$. Suppose there is one, then it must intersect with the line $x_{2}=k a_{12} x_{1}$ at some point, say $x_{0}=\left[\begin{array}{l}x_{01} \\ x_{02}\end{array}\right], x_{02}<1$, then at $x_{0}, \dot{x}_{2}=0$ and $x_{0} \in$ $S_{0}$. When $a_{22}=1, \psi\left(t, x_{0}, a_{22}\right)=\psi_{2}\left(t, x_{0}\right)$ is an ellipse that touches the lines $x_{2}= \pm x_{02}$ (see the solid curve in Fig. 12). By Lemma 5.8, as $a_{22}$ is decreased to $a_{22}-\delta, \psi\left(t, x_{0}, a_{22}-\delta\right)$ will intersect with $\psi\left(t, x_{0}, a_{22}\right)$ at a point to the right of $x_{0}$ and $-x_{0}$. After that, it will stay above $\psi\left(t, x_{0}, a_{22}\right)$ and above the line $x_{2}=-x_{02}$ (see the dashed curves in Fig. 12). Thus no closed trajectory can be formed.

If $k>1$, then $a_{11} a_{21}>a_{12} a_{22}$. If $k=1$, then $a_{11} a_{21}=$ $a_{12} a_{22}$ and it can be verified that $\beta=a_{22}, \beta h_{1}+h_{2}=a_{22}(1-$ $\left.a_{22}\right) h_{1}<0$ (assume $a_{11}=1$ ). So by Proposition 4.1, all the trajectories of (27) are bounded, and they must converge to the origin.

Proof of Theorem 2.1: Combining Remark 2.1, Propositions 3.1, 4.1, and 5.1, we can obtain the necessary and sufficient condition for the system (4) to be GAS. Condition b) in Theorem 2.1 is a simple combination of Condition b) in Remark 2.1 and Propositions 3.1, 4.1, and 5.1. This simplification is justified as follows.

Since $A$ is Hurwitz and $a_{22} \geq 0$, we must have $a_{12}>0$.

If $a_{12} \leq a_{11}$, Remark $2.1 \mathrm{~b}$ ) and Proposition 3.1 say the system is GAS. If $a_{12}>a_{11}$, because $a_{11} a_{21} \geq a_{12} a_{22}$, the system is also GAS by Proposition 5.1.

Conversely, suppose $a_{22} \geq 0$ but $a_{11} a_{21}<a_{12} a_{22}$, we have

$$
\frac{a_{12}}{a_{11}}>\frac{a_{21}}{a_{22}} .
$$

Since $A$ is Hurwitz

$$
\frac{a_{12}}{a_{11}}>\frac{a_{22}}{a_{21}} .
$$


Therefore

$$
\frac{a_{12}}{a_{11}}>\max \left(\frac{a_{21}}{a_{22}}, \frac{a_{22}}{a_{21}}\right) \geq 1
$$

i.e., $a_{12}>a_{11}$. Hence by Propositions 3.1 and 4.1, the system is not GAS whether $a_{21}>a_{22}$ or not. And in both cases, the system has unbounded trajectories and there is also a closed trajectory.

\section{CONCLUSIONS}

We gave a complete stability analysis of a planar linear system under saturation. The analysis involves intricate investigation on the vector field and the intersections of the trajectories with the lines $x_{1}= \pm 1$ and $x_{2}= \pm 1$. Our main result provides a necessary and sufficient condition for such a system to be GAS.

\section{APPENDIX \\ PROOF OF LEMMAS}

\section{Proof of Lemma 5.1}

Under the condition, we have $a_{21}>a_{22}, a_{11} a_{21}>a_{12} a_{22}$. By Proposition 4.1, the system has a bounded global attractor. We need to show that there exists no closed trajectory. Suppose, on the contrary, that there is such a one. Denote the region enclosed by the closed trajectory as $Q$, then by Green's Theorem

$$
\iint_{Q}\left(\frac{\partial f_{1}}{\partial x_{1}}+\frac{\partial f_{2}}{\partial x_{2}}\right) d x_{1} d x_{2}=0
$$

Since a closed trajectory must enclose the origin, the area of the intersection of $Q$ and the central square is nonzero. Thus, the left-side integral is strictly smaller than zero. Note that $\left(\partial f_{1} / \partial x_{1}\right)+\left(\partial f_{2} / \partial x_{2}\right)=-1$ in the central square and nonpositive in other parts of the plane. This is a contradiction. $\square$

\section{Proof of Lemma 5.2}

We only need to show that the lemma is true when $a_{22}=1$. First, let $k=1$, then

$$
h_{1}\left(a_{12}\right)=-2 a_{12}+\left(a_{12}^{2}-1\right) \log \frac{a_{12}+1}{a_{12}-1} .
$$

As $a_{12} \rightarrow 1, h_{1} \rightarrow-2$ and as $a_{12} \rightarrow \infty, h_{1} \rightarrow 0$. Suppose there is an extremum $a_{12}^{*}$ in the interval $(1, \infty)$, then at this extremum $d h_{1} / d a_{12}=0$. From routine computation, this implies

$$
\log \frac{a_{12}^{*}+1}{a_{12}^{*}-1}=\frac{2}{a_{12}^{*}} .
$$

Put this into the formula (28), we get the only possible extremum value

$$
h_{1}\left(a_{12}^{*}\right)=-\frac{2}{a_{12}^{*}}<0
$$

Since $h_{1} \leq 0$ at the two end points of the interval $(1, \infty)$, we must have $h_{1}<0$ on the whole interval.
Next we show that for any fixed $a_{12}>1, h_{1}<0$ for all $k \geq 1$. Here we have

$$
h_{1}(k)=-2 a_{12}+\left(k a_{12}^{2}-1\right) \log \frac{k a_{12}+1}{k a_{12}-1} .
$$

We have just shown that for any given $a_{12}>1, h_{1}(1)<0$. As $k \rightarrow \infty$, we also have $h_{1} \rightarrow 0$. Suppose there is an extremum $k^{*}$ between $(1, \infty)$, then $d h_{1} / d k=0$. This implies

$$
\log \frac{k^{*} a_{12}+1}{k^{*} a_{12}-1}=\frac{2\left(k^{*} a_{12}^{2}-1\right)}{a_{12}\left(k^{*} a_{12}-1\right)\left(k^{*} a_{12}+1\right)}
$$

Put this into the function $h_{1}(k)$, we get

$$
h_{1}\left(k^{*}\right)=\frac{2 a_{12}^{2}+2-4 k^{*} a_{12}^{2}}{a_{12}\left(k^{*} a_{12}-1\right)\left(k^{*} a_{12}+1\right)}<0
$$

(note that $a_{12}>1, k \geq 1$ ). It follows that $h_{1}(k)<0$ for all $k \geq 1$ and $a_{12}>1$.

\section{Proof of Lemma 5.3}

a) This can be shown by comparing $\psi_{2}\left(t, x_{0}\right)$ with $\psi_{1}\left(t, x_{0}\right)$. Since $\psi_{1}\left(t, x_{0}\right)$ is symmetric with respect to the line $x_{1}=x_{2}$, it will intersect with $x_{1}=1$ at $\left[\begin{array}{c}1 \\ x_{01}\end{array}\right]$ for any $x_{01} \in\left[1 / a_{12}, 1\right]$. Since at the same point $x$, if $x_{1}>0$, then $\dot{x}_{2}$ of (25) is smaller (more negative) than that of (26) and $\dot{x}_{1}$ of the two is the same, so $\psi_{2}\left(t, x_{0}\right)$ is below $\psi_{1}\left(t, x_{0}\right)$. Hence, the first intersection of $\psi_{2}\left(t, x_{0}\right)$ with $x_{1}=1$, if there is one, must be below that of $\psi_{1}\left(t, x_{0}\right)$ with $x_{1}=1$, which is $\left[\begin{array}{c}1 \\ x_{01}\end{array}\right]$. This shows that $\left\|x_{0}-\left[\begin{array}{l}1 \\ 1\end{array}\right]\right\|<\left\|x_{c}-\left[\begin{array}{l}1 \\ 1\end{array}\right]\right\|$.

If $x_{01} \in\left[1 / k a_{12}, 1 / a_{12}\right], \psi_{2}\left(t, x_{0}\right)$ will go downward-rightward at first and when it reaches the line $x_{1}=1 / a_{12}$, it is below the point $\left[\begin{array}{c}1 / a_{12} \\ 1\end{array}\right]$. Since $\psi_{1}\left(t,\left[\begin{array}{c}1 / a_{12} \\ 1\end{array}\right]\right)$ does not go beyond the line $x_{1}=1$ (at the intersection $\left.\dot{x}_{1}=0\right), \psi_{2}\left(t, x_{0}\right)$ will not intersect with the line before it turns leftward.

b) If $x_{02}<1 / a_{12}$, then $\psi_{2}\left(t, x_{0}\right)$ goes downward-leftward. Suppose $\psi_{2}\left(t, x_{0}\right)$ intersects with $x_{2}=-1$ at $x_{c}$. Let the region enclosed by $\psi_{2}\left(t, x_{0}\right)$ and the two lines $x_{1}=1$, $x_{2}=-1$ be $S$, then by Green's Theorem

$$
\begin{aligned}
& \oint_{\partial S} f_{2}(x) d x_{1}-f_{1}(x) d x_{2} \\
& =\iint_{S}\left(\frac{\partial f_{1}}{\partial x_{1}}+\frac{\partial f_{2}}{\partial x_{2}}\right) d x_{1} d x_{2} \\
& =0
\end{aligned}
$$

where $\partial S$ denotes the boundary of $S$ that goes clockwise. Note that along the trajectory $\psi_{2}\left(t, x_{0}\right)$, the integral on the boundary is zero, so we have

$$
-\int_{x_{02}}^{-1}\left(-1+a_{12} x_{2}\right) d x_{2}+\int_{1}^{x_{c 1}}\left(-k a_{12} x_{1}-1\right) d x_{1}=0
$$


Let

$$
\begin{aligned}
& c=\left\|x_{c}-\left[\begin{array}{r}
1 \\
-1
\end{array}\right]\right\|=1-x_{c 1} \\
& e=\left\|x_{0}-\left[\begin{array}{r}
1 \\
-1
\end{array}\right]\right\|=x_{02}+1
\end{aligned}
$$

then from the above equation

$\frac{k a_{12}}{2} c^{2}-\left(1+k a_{12}\right) c=\frac{a_{12}}{2} e^{2}-\left(1+a_{12}\right) e$.

Since $\psi_{2}\left(t, x_{0}\right)$ intersects with $x_{2}=-1$ downward, so at $x_{c}$ we must have $\dot{x}_{2} \leq 0$. This implies $x_{c}$ must be to the right of $\left[\begin{array}{c}-\left(1 / k a_{12}\right) \\ -1\end{array}\right]$, i.e., $c \leq 1+1 / k a_{12}$. Similarly, $e \leq 1+1 / a_{12}$. It can be shown by the manipulation of quadratic functions that for any $e \leq 1+1 / a_{12}$ there is a unique $c \leq 1+1 / k a_{12}$ satisfying (29) and $c<e$ due to $k>1$ and $a_{12}>1$.

\section{Proof of Lemma 5.4}

Since $s_{1} \in\left(1 / a_{12}, 1\right]$, at $x_{0}, \dot{x}_{1}>0$, and $\dot{x}_{2}<0$. Thus, $\psi_{2}\left(t, x_{0}\right)$ goes downward-rightward. In the region $W$, above the line $x_{2}=1 / a_{12}, \dot{x}_{1}>0$ and below the line $\dot{x}_{1}<0$, so $\psi_{2}\left(t, x_{0}\right)$ turns downward-leftward on meeting this line.

By the horizontal shifting property (12) of the trajectories in the region $W$, it suffices to show that the lemma is true for one $\gamma>0$. Choose $\gamma$ sufficiently large such that when $\psi_{2}\left(t, x_{0}\right)$ reaches the line $x_{2}=-s_{1}$ at $\left[\begin{array}{c}1+\gamma+\Delta \\ -s_{1}\end{array}\right]$, it is still inside $W$, i.e., $\gamma+\Delta \geq 0$. Obviously, the quantity $\Delta$ is independent of $\gamma$ like $h_{1}$. If $\Delta<0$, then $\left[\begin{array}{c}1+\gamma+\Delta \\ -s_{1}\end{array}\right]$ is to the left of the line $x_{1}=1+\gamma$. This implies $\psi_{2}\left(t, x_{0}\right)$ must have intersected the line $x_{1}=1+\gamma$ at $\left[\begin{array}{c}1+\gamma \\ s_{2}\end{array}\right]$ before it reaches $\left[\begin{array}{c}1+\gamma+\Delta \\ -s_{1}\end{array}\right]$. Note that $\psi_{2}\left(t, x_{0}\right)$ goes downward-leftward below the line $x_{2}=1 / a_{12}$. Thus $s_{2}>-s_{1}$, i.e., $s_{1}+s_{2}>0$.

What remains to be shown is that $\Delta<0$ is indeed the case. Trivial calculation shows that

$$
\Delta=-2 a_{12} s_{1}+\left(k a_{12}^{2}-1\right) \log \frac{k a_{12}+s_{1}}{k a_{12}-s_{1}} .
$$

When $s_{1}=1$ we get $\Delta=h_{1}$. Let $\bar{a}_{12}=a_{12} s_{1}, \bar{k}=k / s_{1}^{2}$, then

$$
\Delta=-2 \bar{a}_{12}+\left(\bar{k} \bar{a}_{12}^{2}-1\right) \log \frac{\bar{k} \bar{a}_{12}+1}{\bar{k} \bar{a}_{12}-1} .
$$

This is similar to $h_{1}$. Since $s_{1} \in\left(1 / a_{12}, 1\right]$, we have $\bar{a}_{12}>$ $1, \bar{k} \geq 1$, so by Lemma $5.2, \Delta<0$.

\section{Proof of Lemma 5.5}

When $x_{01} \geq 1$ we must have $x_{c 1}<x_{01}$, otherwise we would get $h_{1} \geq 0$. However, we know that $h_{1}<0$ by Lemma 5.2. What remains to be shown is the case where $x_{01} \in\left(1 / k a_{12}, 1\right)$. Since $x_{c 1}$ depends continuously on $x_{01}$, it suffices to show that $x_{c 1} \neq x_{01}$ for any $x_{01} \in\left(1 / k a_{12}, 1\right)$. We prove this by contradiction.
Assume that $x_{c 1}=x_{01}$ for some $x_{01} \in\left(1 / k a_{12}, 1\right)$. Then the line $x_{0}$ to $x_{c}$ is vertical.

Case 1: $\psi_{2}\left(t, x_{0}\right)$ does not intersect with the line $x_{1}=1$ before it reaches $x_{2}=-1$. Applying Green's Theorem to the region enclosed by $\psi_{2}\left(t, x_{0}\right)$ and the vertical line $x_{0}$ to $x_{c}$. Since $\partial f_{1} / \partial x_{1}+\partial f_{2} / \partial x_{2}=0$ in the region, we have

$$
\begin{aligned}
\oint f_{2}(x) & d x_{1}-f_{1}(x) d x_{2} \\
& =-\int_{-1}^{1}\left(-x_{01}+a_{12} x_{2}\right) d x_{2}=0 .
\end{aligned}
$$

This leads to $x_{01}=0$, which contradicts the condition that $x_{01}>1 / k a_{12}$.

Case 2: $\psi_{2}\left(t, x_{0}\right)$ intersects with the line $x_{1}=1$ before it reaches $x_{2}=-1$. Let the intersections be $p_{1}=$ $\left[\begin{array}{c}1 \\ s_{1}\end{array}\right], p_{2}=\left[\begin{array}{c}1 \\ s_{2}\end{array}\right]$, see Fig. 8. Again applying Green's Theorem to the region enclosed by the line $x_{c}$ to $x_{0}$, the line $p_{1}$ to $p_{2}$ and the trajectory $\psi_{2}\left(t, x_{0}\right)$, we get

$$
\begin{aligned}
& \int_{-1}^{1}\left(-x_{01}+a_{12} x_{2}\right) d x_{2} \\
& +\int_{s_{1}}^{s_{2}}\left(-1+a_{12} x_{2}\right) d x_{2}=0 .
\end{aligned}
$$

This leads to

$$
2 x_{01}=\left(s_{1}-s_{2}\right)\left(1-\frac{a_{12}}{2}\left(s_{1}+s_{2}\right)\right) \text {. }
$$

By Lemma 5.3, $s_{1} \leq x_{01}, s_{2} \geq-x_{01}$ (= is taken when $k=1$ ), so $s_{1}-s_{2} \leq 2 x_{01}$. By Lemma 5.4, $s_{1}+s_{2}>0$, so $\left(1-\left(a_{12} / 2\right)\left(s_{1}+s_{2}\right)\right)<1$. This contradicts $(30)$.

Combining the two cases, we must have $x_{c 1} \neq x_{01}$ for any $x_{01} \in\left(1 / k a_{12}, 1\right)$. Also, by continuity, $x_{01}>x_{c 1}$.

\section{Proof of Lemma 5.6}

At $x^{*}, \dot{x}_{2}=0, \dot{x}_{1}>0$, so $\psi_{2}\left(t, x^{*}\right)$ goes rightward. By Lemma 5.3, $\psi_{2}\left(t, x^{*}\right)$ will not intersect with the line $x_{1}=1$ before it turns leftward. Since $A$ has a pair of pure imaginary eigenvalues, the trajectory will touch the line $x_{2}=-1$ at $-x^{*}$. And by symmetry, it will return to $x^{*}$ thus form a closed curve. Note that because at $\pm x^{*}, \dot{x}_{2}=0$, so $\psi_{2}\left(t, x^{*}\right)$ has only one intersection with each of the lines $x_{2}=1$ and $x_{2}=-1$. It follows that $\psi_{2}\left(t, x^{*}\right)$ is inside the central square.

If $x_{0} \in S_{0}$, then $\psi_{2}\left(t, x_{0}\right)$ will stay within $S_{0}$ since the trajectories will not intersect with $\psi_{2}\left(t, x^{*}\right)$. Thus $\psi_{2}\left(t, x_{0}\right)$ is in the linear region and will be a closed trajectory.

Since $a_{11} a_{21}=k a_{12}>a_{12}=a_{12} a_{22}, a_{12}>a_{11}$ and $a_{21}>a_{22}$, Condition a) in Proposition 4.1 is satisfied, thus, every trajectory $\psi_{2}\left(t, x_{0}\right)$ of (25) will enter a bounded attractor and hence is bounded. To prove the remaining part of the lemma, it suffices to show that there is no closed trajectory outside $S_{0}$. We prove this by contradiction.

Suppose there is a closed trajectory outside of $S_{0}$, say $\Gamma$, then $\Gamma$ goes clockwise and must have two intersections with the line 


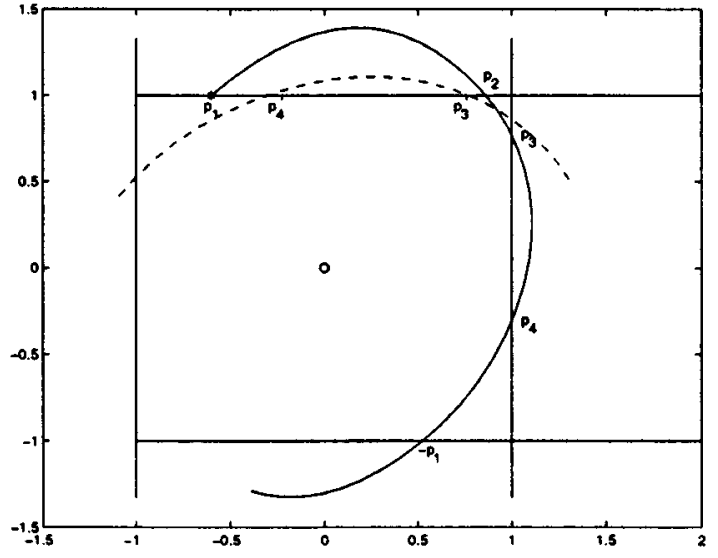

Fig. 13. Illustration for the Proof of Lemma 5.6: Case 1.

$x_{2}=1$. Denote the region enclosed by $\Gamma$ as $S$, then by Green's Theorem, we have

$$
\iint_{S}\left(\frac{\partial f_{1}}{\partial x_{1}}+\frac{\partial f_{2}}{\partial x_{2}}\right) d x_{1} d x_{2}=0
$$

Denote the area of a region $X$ as $\mathcal{A}(X)$, then

$$
\mathcal{A}(S \cap U)-\mathcal{A}(S \cap W)=0 .
$$

Note that in the central square and in the region $V, R$, $\partial f_{1} / \partial x_{1}+\partial f_{2} / \partial x_{2}=0$, in $U, \partial f_{1} / \partial x_{1}+\partial f_{2} / \partial x_{2}=-1$ and in $W, \partial f_{1} / \partial x_{1}+\partial f_{2} / \partial x_{2}=1$. Also note that $S$ must be symmetric with respect to the origin. Equation (31) implies that $\Gamma$ must also intersect with the line $x_{1}=1$ and the area of the part of $S$ in the region $U$ and that in the region $W$ must be equal. We will show that this is impossible.

Case 1: The intersections are all on the boundary of the central square. See Fig. 13, where $p_{1}, p_{2}$ are the intersections with $x_{2}=1, p_{3}, p_{4}$ are the intersections with $x_{1}=1$. By symmetry, $\Gamma$ should intersect with $x_{2}=-1$ at $-p_{1}$. The contradiction will be $\mathcal{A}(S \cap U)>\mathcal{A}(S \cap W)$.

\section{Denote}

$$
p_{1}=\left[\begin{array}{c}
s_{1} \\
1
\end{array}\right], \quad p_{2}=\left[\begin{array}{c}
s_{2} \\
1
\end{array}\right], \quad p_{3}=\left[\begin{array}{c}
1 \\
s_{3}
\end{array}\right], \quad p_{4}=\left[\begin{array}{c}
1 \\
s_{4}
\end{array}\right] .
$$

Then by Lemma 5.3, $s_{3}<s_{2}, s_{4}>-\left(-s_{1}\right)$ and hence

$$
s_{1}<s_{4}<s_{3}<s_{2} \text {. }
$$

Get a symmetric projection of $\Gamma_{p_{3} \rightarrow p_{4}}$ with respect to the line $x_{1}=x_{2}$ on the region $U$ and denote it as $\Gamma_{p_{3} \rightarrow p_{4}}^{\prime}$ (see the dashed curve in Fig. 13). The corresponding intersections with the line $x_{2}=1$ are $p_{3}^{\prime}=\left[\begin{array}{c}s_{3} \\ 1\end{array}\right], p_{4}^{\prime}=\left[\begin{array}{c}s_{4} \\ 1\end{array}\right]$. From (32), $p_{3}^{\prime}$ and $p_{4}^{\prime}$ are between $p_{1}$ and $p_{2}$.

At a point $x i n \Gamma_{p_{1} \rightarrow p_{2}}$, the slope of $\Gamma$ is

$$
\eta_{1}\left(x_{1}\right)=\frac{-k a_{12} x_{1}+1}{-x_{1}+a_{12}} .
$$

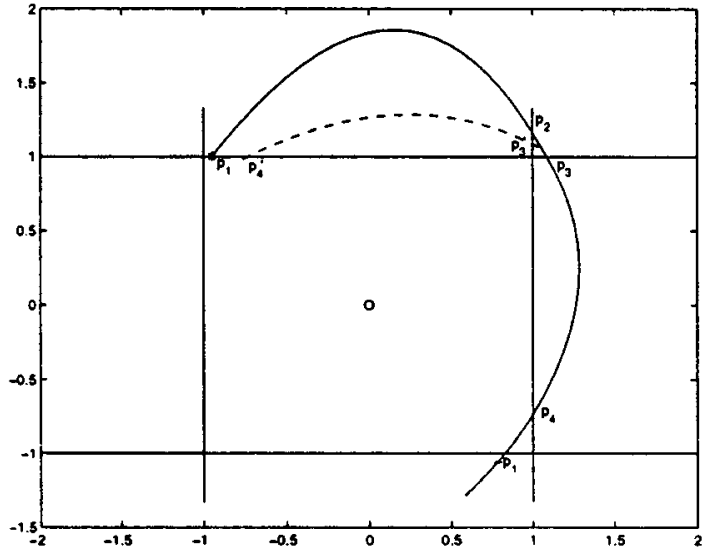

Fig. 14. Ilustration for the Proof of Lemma 5.6: Case 2.

By symmetry (exchanging $x_{1}$ and $x_{2}$ and taking the inverse of the slope), at a point $x \in \Gamma_{p_{3} \rightarrow p_{4}}^{\prime}$ the slope of $\Gamma^{\prime}$ is

$$
\eta_{2}\left(x_{1}\right)=\frac{-1+a_{12} x_{1}}{-k a_{12}+x_{1}} .
$$

Clearly, $\mathcal{A}(S \cap W)$ equals the area of the region enclosed by $\Gamma_{p_{3} \rightarrow p_{4}}^{\prime}$ and the line $x_{2}=1$. For the two areas $\mathcal{A}(S \cap U)$ and $\mathcal{A}(S \cap W)$ to be equal, $\Gamma_{p_{1} \rightarrow p_{2}}$ and $\Gamma_{p_{3} \rightarrow p_{4}}^{\prime}$ must have two intersections, say $x_{l}=\left[\begin{array}{l}x_{11} \\ x_{l 2}\end{array}\right]$ and $x_{r}=\left[\begin{array}{l}x_{21} \\ x_{r 2}\end{array}\right]$, with $x_{l 1}<x_{r 1}$. At the left intersection $x_{l}, \Gamma_{p_{3} \rightarrow p_{4}}^{\prime}$ crosses $\Gamma_{p_{1} \rightarrow p_{2}}$ upward and at the right intersection $x_{r}, \Gamma_{p_{3} \rightarrow p_{4}}^{\prime}$ crosses $\Gamma_{p_{1} \rightarrow p_{2}}$ downward. This implies

$$
\eta_{1}\left(x_{l 1}\right)<\eta_{2}\left(x_{l 1}\right), \quad \eta_{1}\left(x_{r 1}\right)>\eta_{2}\left(x_{r 1}\right)
$$

Let

$$
\begin{aligned}
\eta_{12}\left(x_{1}\right) & =\eta_{1}\left(x_{1}\right)-\eta_{2}\left(x_{1}\right) \\
& =\frac{-a_{12}(k-1)\left(x_{1}^{2}-(k+1) a_{12} x_{1}+1\right)}{\left(-x_{1}+a_{12}\right)\left(-k a_{21}+x_{1}\right)}
\end{aligned}
$$

then $\eta_{12}(-1)>0, \eta_{12}(1)<0$ and from (33), we have

$$
\eta_{12}\left(x_{l 1}\right)<0, \quad \eta_{12}\left(x_{r 1}\right)>0 .
$$

The function changes sign three times, so $\eta_{12}\left(x_{1}\right)$ has at least three zeros between -1 and -1 . Obviously there are only two zeros in this interval, hence, $\Gamma_{p_{3} \rightarrow p_{4}}^{\prime}$ and $\Gamma_{p_{1} \rightarrow p_{2}}$ cannot have two intersections. Consequently, $\mathcal{A}(S \cap U)>\mathcal{A}(S \cap W)$. A contradiction.

Case 2: $\Gamma$ intersects with the lines $x_{1}=1, x_{2}=1$ as in Fig. 14. From $p_{2}$ to $p_{3}$, the slope of the straight line is $\alpha=\left(-k a_{12}+1\right) /\left(-1+a_{12}\right)<-1$, so $\left\|p_{2}-\left[\begin{array}{l}1 \\ 1\end{array}\right]\right\|>\left\|p_{3}-\left[\begin{array}{l}1 \\ 1\end{array}\right]\right\|$. From Lemma 5.3, $\left\|p_{4}-\left[\begin{array}{c}1 \\ -1\end{array}\right]\right\|>$ $\left\|-p_{1}-\left[\begin{array}{l}1 \\ -1\end{array}\right]\right\|=\left\|p_{1}-\left[\begin{array}{c}-1 \\ 1\end{array}\right]\right\|$, note that $\Gamma$ is symmetric and must intersect with $x_{2}=-1$ at $-p_{1}$. Hence, if we get a symmetric projection of $\Gamma_{p_{3} \rightarrow p_{4}}$ on the region $U$ (see the dashed curve in Fig. 14), then $p_{4}^{\prime}$ is to the right of $p_{1}$ and $p_{3}^{\prime}$ is below $p_{2}$. Suppose $\mathcal{A}(S \cap U)=\mathcal{A}(S \cap W), \Gamma_{p_{1} \rightarrow p_{2}}$ will 


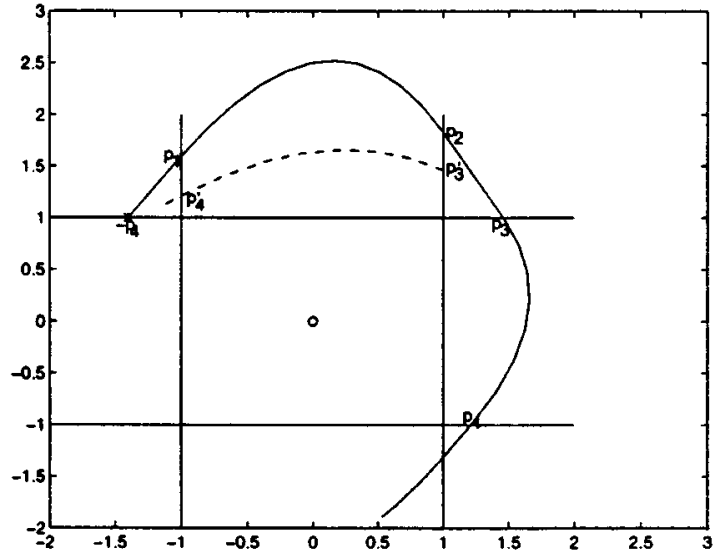

Fig. 15. Illustration for the Proof of Lemma 5.6: Case 3.

intersect with $\Gamma_{p_{3} \rightarrow p_{4}}^{\prime}$ twice. This is impossible as was shown in Case 1.

Case 3: The four intersections are as shown in Fig. 15.

Similarly, since $\alpha<-1, \beta>1$, we have $\left\|p_{2}-\left[\begin{array}{l}1 \\ 1\end{array}\right]\right\|>$ $\left\|p_{3}-\left[\begin{array}{l}1 \\ 1\end{array}\right]\right\|$ and $\left\|p_{4}-\left[\begin{array}{c}1 \\ -1\end{array}\right]\right\|<\left\|-p_{1}-\left[\begin{array}{c}1 \\ -1\end{array}\right]\right\|=$ $\left\|p_{1}-\left[\begin{array}{c}-1 \\ 1\end{array}\right]\right\|$. Also get a symmetric projection of $\Gamma_{p_{3} \rightarrow p_{4}}$ on the region $U$ (see the dashed curve in Fig. 15), then $p_{4}^{\prime}$ is below $p_{1}$ and $p_{3}^{\prime}$ is below $p_{2}$. Similar to Case 1 , we can show that $\mathcal{A}(S \cap U)>\mathcal{A}(S \cap W)$. A contradiction.

Because $h_{1}<0$, there is no such case where $\Gamma$ only encloses $\left[\begin{array}{c}1 \\ -1\end{array}\right]$ and $\left[\begin{array}{c}-1 \\ 1\end{array}\right]$ but not $\left[\begin{array}{l}1 \\ 1\end{array}\right]$ and $\left[\begin{array}{l}-1 \\ -1\end{array}\right]$.

Combining the above three cases, we see that there is no closed trajectory that intersects with any of the lines $x_{1}= \pm 1$ and $x_{2}= \pm 1$ twice. So there is no closed trajectory outside of $S_{0}$ and if a trajectory starts outside of $S_{0}$, it will converge to the boundary of $S_{0}$.

\section{Proof of Lemma 5.7}

Since $x_{0}$ is outside of $S_{0}$ and is to the left of $x^{*}$, so $\psi_{2}\left(t, x_{0}\right)$ goes upward-rightward at first. After crossing the line $x_{1}=$ $1 / k a_{12}$, it goes downward and returns to the line $x_{2}=1$. Since $\psi_{2}\left(t, x_{0}\right)$ goes clockwise, at the first intersection with the line $x_{2}=-1$ it crosses the line downward, so $\dot{x}_{2}<0$ at $x_{c}$ and $x_{c 1}>-1 / k a_{12}$. Let $t_{m}$ be the time when $\psi_{2}\left(t, x_{0}\right)$ intersects with $x_{2}=-1$, i.e., $\psi_{2}\left(t_{m}, x_{0}\right)=x_{c}$. Suppose $x_{c 1}=-x_{01}$, then $x_{c}=-x_{0}$ and $\left\{\psi_{2}\left(t, x_{0}\right), t \in\left[0,2 t_{m}\right]\right\}$ is a closed curve. This is impossible by Lemma 5.6. Now suppose $x_{c 1}>-x_{01}$, then $x_{c}$ is to the right of $-x_{0}$. Let the region enclosed by $\left\{\psi_{2}\left(t, x_{0}\right), t \in\left[0, t_{m}\right]\right\},\left\{\psi_{2}\left(t,-x_{0}\right), t \in\left[0, t_{m}\right]\right\}$, the line $x_{c}$ to $-x_{0}$ and the line $-x_{c}$ to $x_{0}$ be $S$, then on the line from $x_{c}$ to $-x_{0}, \dot{x}_{2}<0$ and $\dot{x}$ points outward from $S$. Similarly, on the line from $-x_{c}$ to $x_{0}, \dot{x}$ also points outward from $S$. Thus, no trajectory outside of $S$ will enter it. This contradicts with Lemma 5.6 since $S_{0}$ is in the interior of $S$.

Therefore, we must have $-\left(1 / k a_{12}\right)<x_{c 1}<-x_{01}$.

\section{Proof of Lemma 5.8}

Without loss of generality, assume $x_{02}=1$. When $x_{02} \in$ $(0,1)$, the proof can be carried out similarly. There are three cases.

Case 1: $x_{01}<1$ and $\psi\left(t, x_{0}, a_{22}\right)$ does not intersect with the line $x_{1}=1$ (see Fig. 16).

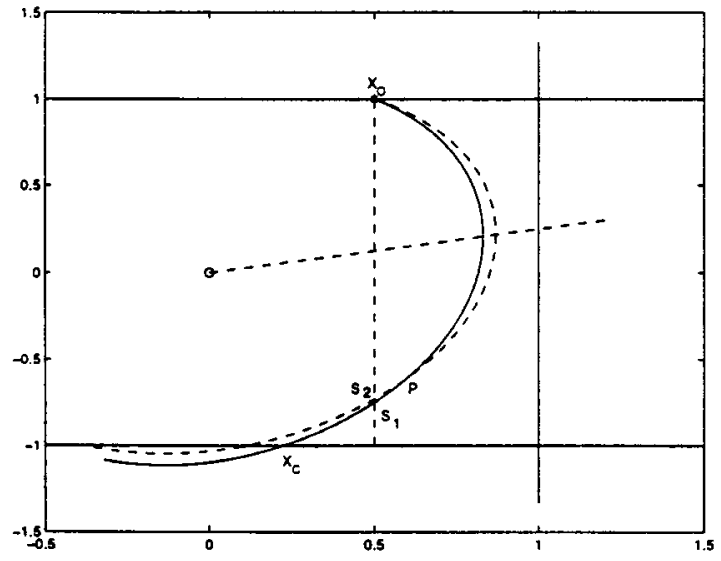

Fig. 16. Illustration for the Proof of Lemma 5.8: Case 1.

Because $x_{01} \geq 1 / k a_{12}$, both $\psi\left(t, x_{0}, a_{22}\right)$ and $\psi\left(t, x_{0}, a_{22}-\delta\right)$ go downward-rightward until reaching the line $x_{2}=x_{1} / a_{12}$ (see the dashed line passing through the origin). (On this line, $\dot{x}_{1}=0$ ). After that, the trajectories turn leftward.

At $t=0$, the slopes of both trajectories are negative and $\eta\left(x_{0}, a_{22}\right)<\eta\left(x_{0}, a_{22}-\delta\right)$, so $\psi\left(t, x_{0}, a_{22}-\delta\right)$ will go to the right of $\psi\left(t, x_{0}, a_{22}\right)$ at the begining. On the part of $\psi\left(t, x_{0}, a_{22}\right)$ that is above the line $x_{2}=x_{1} / a_{12}, \eta\left(x, a_{22}\right)<$ $\eta\left(x, a_{22}-\delta\right)$ and on the part that is below the line $\eta\left(x, a_{22}\right)>$ $\eta\left(x, a_{22}-\delta\right)$. So $\psi\left(t, x_{0}, a_{22}-\delta\right)$ can only cross $\psi\left(t, x_{0}, a_{22}\right)$ leftward below the line. We will show that the crossing point $p$ (see Fig. 16) is to the right of $x_{0}$ and $x_{c}$. After the crossing, $\psi\left(t, x_{0}, a_{22}-\delta\right)$ will stay to the left of $\psi\left(t, x_{0}, a_{22}\right)$ until meeting the line $x_{2}=-1$. This leads to the desired result.

Let $s_{1}=\left[\begin{array}{c}x_{01} \\ e_{1}\end{array}\right], s_{2}=\left[\begin{array}{c}x_{01} \\ e_{2}\end{array}\right]$ be the intersections of $\psi\left(t, x_{0}, a_{22}\right)$ and $\psi\left(t, x_{0}, a_{22}-\delta\right)$ with the vertical line $x_{1}=x_{01}$, respectively. Since $x_{c}$ is to the left of $x_{0}$, so $s_{1}$ is above the line $x_{2}=-1$. Assume on the contrary that there is no intersection of $\psi\left(t, x_{0}, a_{22}-\delta\right)$ with $\psi\left(t, x_{0}, a_{22}\right)$ that is to the right of the line $x_{1}=x_{01}$, then $s_{1}$ must be above $s_{2}$, i.e., $e_{1}>e_{2}$ and $\psi\left(t, x_{0}, a_{22}-\delta\right)$ is to the right of $\psi\left(t, x_{0}, a_{22}\right)$ before meeting the line $x_{1}=x_{01}$. Denote the area of the region enclosed by $\psi\left(t, x_{0}, a_{22}\right)$ and the line from $x_{0}$ to $s_{1}$ as $\mathcal{A}$ and the area of the region enclosed by $\psi\left(t, x_{0}, a_{22}-\delta\right)$ with the line from $x_{0}$ to $s_{2}$ as $\mathcal{B}$, then $\mathcal{A}<\mathcal{B}$. Applying Green's theorem to the vector fields corresponding to $a_{22}$ and $a_{22}-\delta$, we have

$$
\begin{aligned}
-\int_{e_{1}}^{1} f_{1}(x) d x_{2} & =\mathcal{A}\left(a_{22}-1\right) \\
-\int_{e_{2}}^{1} f_{1}(x) d x_{2} & =\mathcal{B}\left(a_{22}-\delta-1\right) .
\end{aligned}
$$

Note that $f_{1}(x)=-x_{1}+a_{12} x_{2}$ is the same for both the vector fields. Subtracting (35) from (34), we obtain

$$
\int_{e_{2}}^{e_{1}} f_{1}(x) d x_{2}=(\mathcal{B}-\mathcal{A})\left(1-a_{22}\right)+\mathcal{B} \delta>0 .
$$

We know that $f_{1}(x)<0$ from $s_{1}$ to $s_{2}$ since the trajectories go leftward. By assumption, $e_{1}>e_{2}$, so $\int_{e_{2}}^{e_{1}} f_{1}(x) d x_{2}<0$. A contradiction. Therefore, we must have $e_{1}<e_{2}$ and 


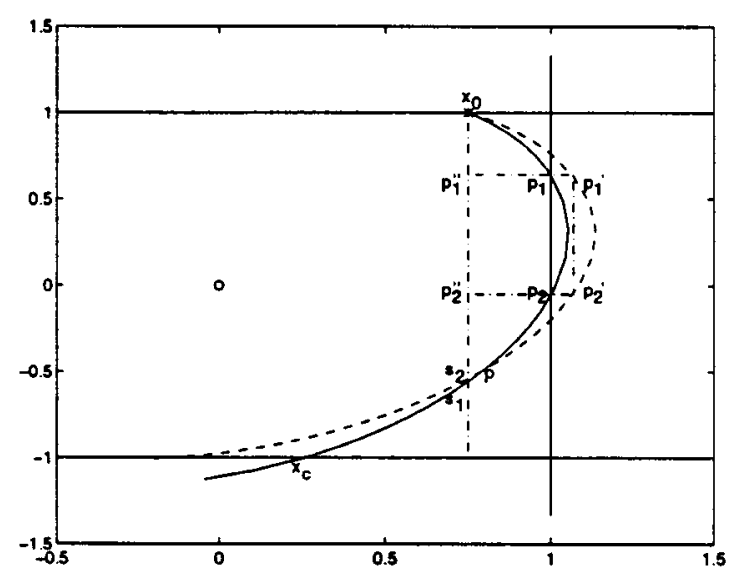

Fig. 17. Illustration for the Proof of Lemma 5.8: Case 2.

$\psi\left(t, x_{0}, a_{22}-\delta\right)$ intersects $\psi\left(t, x_{0}, a_{22}\right)$ at a point $p$ to the right of $x_{0}$.

Case 2: $x_{01}<1$ and $\psi\left(t, x_{0}, a_{22}\right)$ intersects with $x_{1}=1$, see Fig. 17.

Let $p_{1}$ be the first intersection of $\psi\left(t, x_{0}, a_{22}\right)$ with $x_{1}=1$, $p_{2}$ be the second one.

Let the horizontal distance from $\psi\left(t, x_{0}, a_{22}\right)$ to $\psi\left(t, x_{0}, a_{22}-\delta\right)$ at $p_{1}$ be $\Delta_{1}$ and that at $p_{2}$ be $\Delta_{2}$. Then $p_{1}+\left[\begin{array}{c}\Delta_{1} \\ 0\end{array}\right]=: p_{1}^{\prime}$ is on $\psi\left(t, x_{0}, a_{22}-\delta\right)$. By the horizontal shifting property (12) of the trajectories, $\psi\left(t, p_{1}^{\prime}, a_{22}\right)$ will intersect the line $x_{1}=1+\Delta_{1}$ at $p_{2}+\left[\begin{array}{c}\Delta_{1} \\ 0\end{array}\right]=: p_{2}^{\prime}$. From Fact 5.1, $\psi\left(t, p_{1}^{\prime}, a_{22}-\delta\right)$ also returns to the line $x_{1}=1+\Delta_{1}$ at $p_{2}^{\prime}$. Because $p_{1}^{\prime}$ is on $\psi\left(t, x_{0}, a_{22}-\delta\right), \psi\left(t, p_{1}^{\prime}, a_{22}-\delta\right)$ overlaps with $\psi\left(t, x_{0}, a_{22}-\delta\right)$. It follows that $\Delta_{2}=\Delta_{1}$.

Let $s_{1}, s_{2}$ and $p$ be defined similarly to Case 1 , we will also show that $s_{2}$ is above $s_{1}$ by contradiction. First, we need an upper bound for $\Delta_{1}$.

Let $v=\left[\begin{array}{l}v_{1} \\ v_{2}\end{array}\right]$ be a point on $\psi\left(t, x_{0}, a_{22}\right)$ and $\left[\begin{array}{c}v_{1}+\Delta \\ v_{2}\end{array}\right]$ be a point on $\psi\left(t, x_{0}, a_{22}-\delta\right)$. Define

$$
g\left(v_{1}, v_{2}\right):=\frac{\left(-k a_{12} v_{1}+v_{2}\right)}{-v_{1}+a_{12} v_{2}}
$$

then the slope of $\psi\left(t, x_{0}, a_{22}\right)$ at $v$ is $a_{22} g\left(v_{1}, v_{2}\right)=: \eta_{1}$ and the slope of $\psi\left(t, x_{0}, a_{22}-\delta\right)$ at $\left[\begin{array}{c}v_{1}+\Delta \\ v_{2}\end{array}\right]$ is $\left(a_{22}-\delta\right) g\left(v_{1}+\right.$ $\left.\Delta, v_{2}\right)=: \eta_{2}$. It easily can be verified that for $v_{2}>0, g\left(v_{1}, v_{2}\right)$ is a decreasing function of $v_{1}$, so

$$
g\left(v_{1}, v_{2}\right)>g\left(v_{1}+\Delta, v_{2}\right), \quad \forall \Delta>0, v_{2}>0
$$

We can view $\Delta$ as a function of $v_{1}$. Routine analysis shows that

$$
\frac{d \Delta}{d v_{1}}=\frac{\eta_{1}-\eta_{2}}{\eta_{2}}
$$

Note that the part of $\psi\left(t, x_{0}, a_{22}\right)$ from $x_{0}$ to $p_{1}$ is above the line $x_{2}=0$. It follows from (36) that

$$
\begin{aligned}
\eta_{1}-\eta_{2} & =a_{22} g\left(v_{1}, v_{2}\right)-\left(a_{22}-\delta\right) g\left(v_{1}+\Delta, v_{2}\right) \\
& =a_{22}\left(g\left(v_{1}, v_{2}\right)-g\left(v_{1}+\Delta, v_{2}\right)\right)+\delta g\left(v_{1}+\Delta, v_{2}\right) \\
& >\delta g\left(v_{1}+\Delta, v_{2}\right)
\end{aligned}
$$

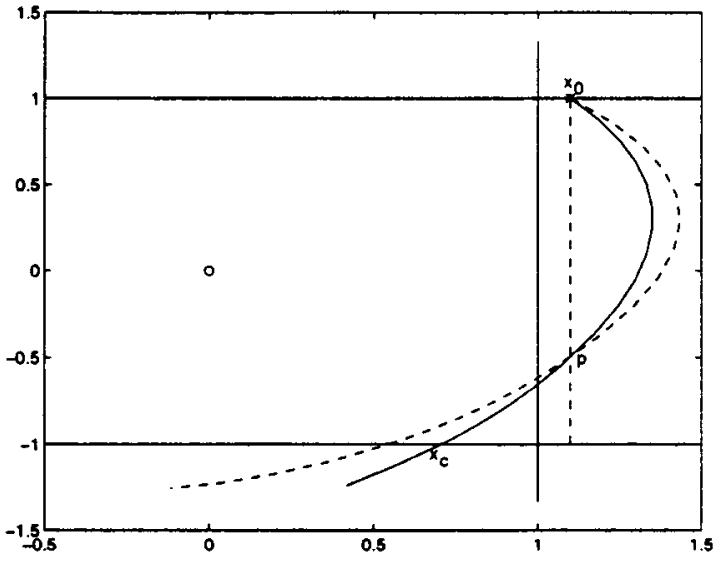

Fig. 18. Illustration for the Proof of Lemma 5.8: Case 3.

Since $g\left(v_{1}+\Delta, v_{2}\right)<0$, we have

$$
\frac{d \Delta}{d v_{1}}<\frac{\delta}{a_{22}-\delta}
$$

Therefore

$$
\Delta_{1}=\int_{x_{01}}^{1} \frac{d \Delta}{d v_{1}} d v_{1}<\frac{\delta}{a_{22}-\delta}\left(1-x_{01}\right) .
$$

Let $p_{1}^{\prime \prime}$ be the intersection of the extension of the line from $p_{1}$ to $p_{1}^{\prime}$ with $x_{1}=x_{01}$ and $p_{2}^{\prime \prime}$ be that of the line from $p_{2}$ to $p_{2}^{\prime}$ (see Fig. 17). Denote $\mathcal{A}_{1}, \mathcal{A}_{2}, \mathcal{A}_{3}, \mathcal{A}_{4}$ as the areas of the regions enclosed by straight lines and $\psi\left(t, x_{0}, a_{22}\right)$ corresponding to the sets of vertices $\left\{x_{0}, p_{1}^{\prime \prime}, p_{1}\right\},\left\{p_{2}^{\prime \prime}, p_{2}, s_{1}\right\},\left\{p_{1}^{\prime \prime}, p_{1}, p_{2}, p_{2}^{\prime \prime}\right\},\left\{p_{1}, p_{2}\right\}$

respectively. Denote $\mathcal{B}_{1}, \mathcal{B}_{2}, \mathcal{B}_{4}, \mathcal{B}_{5}$ as the areas of the regions enclosed by straight lines and $\psi\left(t, x_{0}, a_{22}-\delta\right)$ corresponding to the sets of vertices $\left\{x_{0}, p_{1}^{\prime \prime}, p_{1}^{\prime}\right\},\left\{p_{2}^{\prime \prime}, p_{2}^{\prime}, s_{2}\right\},\left\{p_{1}^{\prime}, p_{2}^{\prime}\right\},\left\{p_{1}, p_{1}^{\prime}, p_{2}^{\prime}, p_{2}\right\}$, respectively. Then by Green's theorem, we have

$$
\begin{aligned}
-\int_{e_{1}}^{1} f_{1}(x) d x_{2}= & \left(-1+a_{22}\right)\left(\mathcal{A}_{1}+\mathcal{A}_{2}\right)+\left(-1+a_{22}\right) \mathcal{A}_{3} \\
& +a_{22} \mathcal{A}_{4} \\
-\int_{e_{2}}^{1} f_{1}(x) d x_{2}= & \left(-1+a_{22}-\delta\right)\left(\mathcal{B}_{1}+\mathcal{B}_{2}\right) \\
& +\left(-1+a_{22}-\delta\right) \mathcal{A}_{3}+\left(a_{22}-\delta\right) \mathcal{B}_{4} \\
& +\left(a_{22}-\delta\right) \mathcal{B}_{5} .
\end{aligned}
$$

Note that there are small triangle areas in $\mathcal{B}_{1}$ and $\mathcal{B}_{2}$ that are in the region $W$. They are of the order $\delta^{2}$. Since $\delta$ is arbitrarily small, we can treat them as a region in the central square for simplicity.

It follows from Fact $5.1 \mathrm{~b}$ ) and the horizontal shifting property in the region $W$ that $a_{22} \mathcal{A}_{4}=\left(a_{22}-\delta\right) \mathcal{B}_{4}$. From (37), we have

$$
\begin{aligned}
& \left(-1+a_{22}\right) \mathcal{A}_{3}-\left(-1+a_{22}-\delta\right) \mathcal{A}_{3}-\left(a_{22}-\delta\right) \mathcal{B}_{5} \\
& \quad=\delta \mathcal{A}_{3}-\left(a_{22}-\delta\right) \mathcal{B}_{5} \\
& \quad=\delta\left\|p_{1}-p_{2}\right\|\left(1-x_{01}\right)-\left(a_{22}-\delta\right)\left\|p_{1}-p_{2}\right\| \Delta_{1}>0
\end{aligned}
$$


By assumption, $s_{1}$ is above $s_{2}$, so $\mathcal{A}_{1}+\mathcal{A}_{2}<\mathcal{B}_{1}+\mathcal{B}_{2}$. Subtracting (39) from (38) we get

$$
\int_{e_{2}}^{e_{1}} f_{1}(x) d x_{2}>0 .
$$

A contradiction with $e_{1}>e_{2}$ and $f_{1}(x)<0$.

Case 3: $x_{01} \geq 1$ (see Fig. 18).

In this case, $\psi\left(t, x_{0}, a_{22}\right)$ goes downward-rightward, then turns downward-leftward and returns to the line $x_{1}=x_{01}$ at a point, say, $p=\left[\begin{array}{l}x_{01} \\ x_{p 2}\end{array}\right]$. Because $h_{1}<0, p$ is above the line $x_{2}=-1$. By Fact $5.1, \psi\left(t, x_{0}, a_{22}-\delta\right)$ will also return to the line $x_{1}=x_{01}$ at the same point $p$. After that , $\psi\left(t, x_{0}, a_{22}-\delta\right)$ remains to the left of $\psi\left(t, x_{0}, a_{22}\right)$ untill it meets the line $x_{2}=$ -1 and the desired result follows.

\section{REFERENCES}

[1] F. Albertini and D. D'Aless, "Asymptotic stability of continuous-time systems with saturation nonlinearities," Syst. Contr. Lett., vol. 29, pp. 175-180, Nov. 1996.

[2] D. S. Bernstein and A. N. Michel, "A chronological bibliography on saturating actuators," Int. J. Robust Nonlinear Contr., vol. 5, pp. 375-380, Aug. 1995.

[3] L. Hou and A. N. Michel, "Asymptotic stability of systems with saturation constraints," IEEE Trans. Automat. Contr., vol. 43, pp. 1148-1154, Aug. 1998.

[4] L. Jin, P. N. Nikiforuk, and M. M. Gupta, "Absolute stability conditions for discrete-time recurrent neural networks," IEEE Trans. Neural Networks, vol. 5, pp. 954-964, Nov. 1994.

[5] Z. Lin, Low Gain Feedback, ser. Lecture Notes in Control and Information Sciences. London, U.K.: Springer-Verlag, 1998.

[6] D. Liu and A. N. Michel, "Asymptotic stability of systems operating on a closed hypercube," Syst. Contr. Lett., vol. 19, pp. 281-285, Oct. 1992.

[7] _ _ "Sparsely interconnected neural networks for associative memories with applications to cellular neural networks," IEEE Trans. Circuits Syst., vol. 41, pp. 295-307, Apr. 1994.

[8] - Dynamical Systems with Saturation Nonlinearities, ser. Lecture Notes in Control and Information Sciences. London, U.K.: Springer, 1994.
[9] R. Mantri, A. Saberi, and V. Venkatasubramanian, "Stability analysis of continuous time planar systems with state saturation nonlinearity," IEEE Trans. Circuits Syst. I, vol. 45, pp. 989-993, Sept. 1998.

[10] J. H. F. Ritzerfeld, "A condition for the overflow stability of secondorder digital filters that is satisfied by all scaled state-space structures using saturation," IEEE Trans. Circuits Syst., vol. 36, pp. 1049-1057, Aug. 1989.

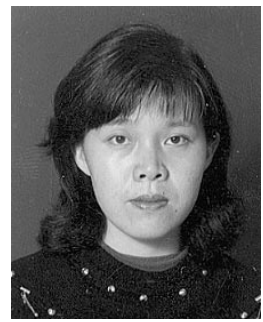

Tingshu Hu (S'99) was born in Sichuan, China, in 1966. She received the B.S. and M.S. degrees in electrical engineering from Shanghai Jiao Tong University, Shanghai, China, in 1985 and 1988 respectively.

She is currently working toward a Ph.D. degree at the Department of Electrical Engineering, University of Virginia, Charlottesville. Her research interests include systems with saturation nonlinearities and robust control theory.

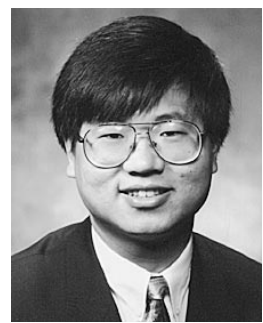

Zongli Lin (S'89-M'90-SM'98) was born in Fuqing, Fujian, China, on February 24, 1964. He received the B.S. degree in mathematics and computer science from Amoy University, Xiamen, China, in 1983, the M.Eng. degree in automatic control from the Chinese Academy of Space Technology, Beijing, China, in 1989, and the Ph.D. degree in electrical and computer engineering from Washington State University, Pullman, WA, in May 1994.

From July 1983 to July 1986, He worked as a Control Engineer at the Chinese Academy of Space Technology. In January 1994 he joined the Department of Applied Mathematics and Statistics, State University of New York at Stony Brook, as a Visiting Assistant Professor. He is now an Assistant Professor in Electrical Engineering at the University of Virginia, Charlottesville. His current research interests include nonlinear control, robust control, and control of systems with saturating actuators. In these areas he has published several papers. He is also the Author of the recent book, Low Gain Feedback (London, U.K.: Springer-Verlag, 1998).

Dr. Lin currently serves as an Associate Editor on the Conference Editorial Board of the IEEE Control Systems Society. He is the Recipient of an ONR Young Investigator Award. 